\title{
Electrokinetic flows through a parallel-plate channel with slipping stripes on walls
}

\author{
Chiu-On $\mathrm{Ng}^{\text {a) }}$ and Henry C. W. Chu \\ Department of Mechanical Engineering, The University of Hong Kong, Pokfulam Road, Hong Kong
}

(Received 11 May 2011; accepted 7 September 2011; published online 14 October 2011)

\begin{abstract}
Longitudinal and transverse electrohydrodynamic flows through a plane channel, of which the walls are micropatterned with a periodic array of stripes, are considered. One unit of wall pattern consists of a slipping stripe and a non-slipping stripe, each with a distinct zeta potential. The problems are solved by a semi-analytical method, where the basic solutions satisfying the electrohydrodynamic equations are expressed by eigenfunction expansions, and the coefficients are determined numerically by point collocation satisfying the mixed stick-slip boundary conditions. In the regime of linear response, the Onsager relations for the fluid and current fluxes are deduced as linear functions of the hydrodynamic and electric forcings. The phenomenological coefficients are explicitly expressed as functions of the channel height, the Debye parameter, the slipping area fraction of the wall, the intrinsic slip length, and the zeta potentials. Attention is paid to some particular kinds of patterns, with a view to revisit and to generalize the theoretical limits made in previous studies on electrokinetic flow over an inhomogeneously slipping surface. One should be cautious when applying the theoretical limits. We show that when a surface is not $100 \%$ uniformly slipping but has a small fraction of area being covered by no-slip slots, the electro-osmotic enhancement can be appreciably reduced. We also show that when the electric double layer is only moderately thin, slipping-uncharged regions on a surface will have finite inhibition effect on the electro-osmotic flow. (C) 2011 American Institute of Physics. [doi:10.1063/1.3647582]
\end{abstract}

\section{INTRODUCTION}

Much of the microfluidic technology relies on flow through a channel with very fine cross-section. Experiments in past decades have explored fluid flows in ever-smaller channels, which can now be miniaturized to microns or even smaller in cross-section. There is an increasing need to look for effective ways to drive fluids through such capillaries. The flow-rate for pressure-driven (also called Poiseuille) flow through a rectangular channel decreases according to the third power of the channel height, and hence pressure pumping alone may be insufficient for very narrow channels. If the fluid is an electrolyte solution (e.g., water with dissolved ionic species or a liquid with free charges), flow may result from electrokinetic phenomena as well. A common kind of such flow, termed electroosmotic flow (EOF), is driven by an electric potential difference across the channel. EOF has typically a plug-like velocity profile, which is determined by the electrical properties but not the channel height.

Electrokinetic phenomena stem from the distribution of free charges in the electrical double layer (EDL) that forms on the fluid-solid interface. Most substances acquire a surface electric charge when brought into contact with an electrolyte. Ions of opposite charge (counterions) to that of the surface will be attracted to the surface, while ions of the same charge (coions) are repelled from the surface. Such a redistribution of free ions in the solution, together with the surface charge, gives rise to a two-layer structure: a single

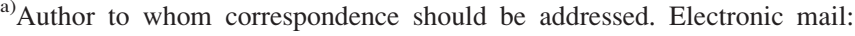
cong@hku.hk.
}

(stern) layer of immobile ions attached to the surface, and a diffuse layer in which there is an excess of counterions over coions to neutralize the surface charge. The electric potential on the stern interface is known as the zeta potential. Charge neutrality is not satisfied in the double layer, and hence, in the presence of an electric field, these ions will move, generating electric current in the channel. At the same time, the electric body force will drive the bulk fluid into motion as well. Such a phenomenon is called electro-osmosis. If the channel is closed, electro-osmotic pressure will be induced to produce a counterbalancing flow. The converse of electroosmosis is known as streaming potential, which is the electric potential induced in a pressure-driven flow in order to produce a counterbalancing electric current when there is no external electric connection between the inlet and exit of the channel. Streaming potential thereby produces a backward flow known as electroviscous flow. Probstein, ${ }^{1} \mathrm{Li}^{2}{ }^{2} \mathrm{Kandli}-$ kar et al., ${ }^{3}$ and Masliyah and Bhattacharjee ${ }^{4}$ can be consulted for an in-depth understanding of electrokinetic transport phenomena.

Boundary slip plays an equally important role in facilitating flow through a microchannel. Microengineered surfaces exhibiting slip length (which corresponds to the depth into the envelope of a surface where a simple shear flow velocity would extrapolate to zero) as large as tens of microns have been reported. Current understanding has the hydrodynamic slip be classified into intrinsic slip and roughnessinduced slip. Intrinsic slip arises from a depletion or lowdensity region separating molecules of fluid from those of a solvophobic surface. Roughness-induced slip, which is an apparent effect as is observed from outside the boundary, 
results from the possible trapping of nano- or micro-bubbles of gas in crevices of a rough surface. Either a low-density region or a layer of gas pockets will appreciably lubricate the flow owing to their much lower viscosity. Studies on superhydrophobic slip flows abound in the literature; see Rothstein ${ }^{5}$ for a most recent review on this topic.

While electrokinetics and hydrodynamics slip are individually beneficial to microchannel flow, the aggregate effects due to their combined action remain fraught with questions. Churaev et al. ${ }^{6}$ experimentally observed the effect of surface slippage on the zeta potential. In the DebyeHückel limit for low potential, they put forward a linear expression for the corrected zeta potential $\zeta_{a} / \zeta=1+\lambda \kappa$, where $\zeta_{a}$ and $\zeta$ are the apparent and true zeta potentials, respectively, $\lambda$ is the slip length of the surface, and $\kappa^{-1}$ is the Debye length that scales the thickness of the electric double layer. Since the EDL is of the order of nanometers, even a small slip length of the order of tens of nanometers can lead to a significant enhancement in the zeta potential. The EOF slip velocity, known as Helmholtz-Smoluchowski velocity, is linearly proportional to the zeta potential and will therefore be enhanced accordingly. Nonlinear relationships for the apparent potential, which work beyond the DebyeHückel limit, were later proposed by Joly et $a l^{7}$ and Tandon and Kirby. ${ }^{8}$

Using the Lorentz reciprocal theorem, Squires ${ }^{9}$ derived a general expression for the electro-osmotic slip velocity, in the thin double-layer limit, for a microscopically heterogeneous surface, where the zeta potential and slip length are given functions of the microscales. His results indicate that (i) the linear enhancement due to slip is only true when the zeta potential is everywhere constant; (ii) electro-osmotic flow over a surface with zero-potential and perfect-slip regions shows no manifestation of the slip at all. In conclusion, Squires remarked that electrokinetic effects over inhomogeneously slipping surfaces depend sensitively on the microscopic nature of the surface phenomena.

Motivated by the findings of Squires, ${ }^{9}$ we aim to further look into the problem of electrokinetic flow over an inhomogeneously slipping and charged surface by considering specifically the pattern of linear stripes aligned either parallel or normal to the flow. We shall re-examine the two observations made by Squires ${ }^{9}$ mentioned above, while relaxing some of the conditions imposed by him. In this work, we shall first derive the Onsager constitutive equations governing the fluid and current fluxes as linear functions of the hydrodynamic and electro-osmotic forcings that are in a direction either parallel or perpendicular to the stripes. Assuming low potentials, the linearized Poisson-Boltzmann equation is used to describe the electric potential as a function of space subject to the periodic change in the zeta potential on the walls. The Boltzmann equation is not applicable when the EDLs of the two walls overlap each other since the conditions of zero electrical potential and ionic concentrations equal to the bulk ionic concentration at a location far away from the walls, which are required for the validity of the Boltzmann equation, will not be satisfied for overlapped EDL fields. ${ }^{10,11}$ Here, we assume that the electric double layer is of a thickness equal to or smaller than the channel height, and therefore only weakly or non-overlapped EDL fields are considered. We also assume that the slipping regions can have a finite or infinite slip length. We solve the flow problems using the method of eigenfunction expansions, where the coefficients are numerically determined by the method of point collocation satisfying the mixed stick and slip conditions on the walls.

The problem under consideration, i.e., electro-osmotic (EO) flow over a striped superhydrophobic surface, has also been studied by Bahga et al., ${ }^{12}$ Zhao, ${ }^{13,14}$ and Belyaev and Vinogradova. ${ }^{15}$ These studies have contributed significantly to the understanding of the effects of an inhomogeneously charged superhydrophobic surface on the EO flow. Relations between EO mobility and hydrodynamic slip have been developed by these authors, particularly for the limits of thin and thick EDL. Conditions under which the EO flow is to be enhanced by hydrodynamic slip are also discussed by these authors. Nevertheless, some generalizations of the problem are still wanting. Most of these studies are confined to flow over a single surface or flow through a thick channel and stripes which are perfect slipping. Here, we consider flow through a channel of any height (subject to the constraint that the EDLs are not overlapped), and stripes of arbitrary slippage. Zhao ${ }^{14}$ has numerically demonstrated the applicability of the Onsager relation for EO flow of arbitrary EDL thickness over a superhydrophobic surface. Here, we can prove it formally. Also, these studies have developed analytical expressions for the thin EDL limit, but the range of validity of these limiting expressions is yet to be ascertained.

These authors used the dual series to solve the mixed boundary value problem. Here, we use the method of point collocation, which is simpler to implement numerically than the dual series. We shall show how our model can produce the same theoretical expressions as those derived by these authors $^{9,12,14,15}$ when applied to some particular kinds of slip-charge wall patterns. First, when the wall potential is uniform, the effective hydrodynamic slip length can be directly employed in the EO flow problem as if the wall were homogeneously slipping. An important implication is that the EO enhancement due to slip is linearly proportional to the ratio of the effective slip length to the electric double layer thickness. Second, when the slip regions are perfectly slipping but uncharged, they will have no effect on the EO flow under the condition of a very thin electric double layer. Third, when the slip regions are partially slipping and uncharged, the EO flow will always be inhibited by the effective slip. However, this trend can be dramatically reversed when the slipping regions become slightly charged. In this paper, we put the emphasis also on how one should be watchful on applying these theoretical limits. We demonstrate our points with numerical results. First, one is cautioned that when a surface is not $100 \%$ uniformly slipping but has a small fraction of area being covered by interspersed no-slip slots, the EO enhancement can be materially reduced. Second, one is cautioned that unless the electric double layer is extremely thin, at least three orders of magnitude thinner than the pattern length, the slipping-uncharged regions will indeed have finite effect (not enhancement, but diminishing) on the EO flow. Further to these two points, we shall also 
examine in this paper how the present wall pattern may have effect on the so-called figure of merit of the channel when it is used for electrokinetic energy conversion.

\section{FORMULATION}

Consider Stokes flow of a fluid through a parallel-plate channel, of which the walls are patterned with a periodic array of alternate stick-slip stripes of different wall potentials. As shown in Fig. 1, the two walls are located at $y= \pm h$, where $h$ is half the channel height. The two walls have identical patterns which are aligned in-phase with each other such that the flow is symmetrical about the $x$-axis. One unit of the wall pattern, which is of a period length $2 L$, consists of a slipping stripe of width $2 a L$, slip length $\lambda$, and wall potential $\zeta_{\mathrm{S}}$ and a non-slipping stripe of width $2(1-a) L$ and wall potential $\zeta_{\mathrm{NS}}$. The number $0 \leq a \leq 1$ denotes the area fraction of the wall which is slipping. The intrinsic or microscopic slip length is allowed to vary in the range $0 \leq \lambda \leq \infty$, where the two limiting values $\lambda=0$ and $\lambda=\infty$ correspond to no-slip and perfectslip conditions, respectively. The pattern period length is assumed to be comparable to the channel height, $L \sim h$.

On assuming low potentials and non-overlapped EDLs, we invoke the Debye-Hückel approximation, by which the Poisson-Boltzmann equation for the electric potential $\psi(x, y)$ due to the electric double layer is linearized to

$$
\nabla^{2} \psi=\kappa^{2} \psi
$$

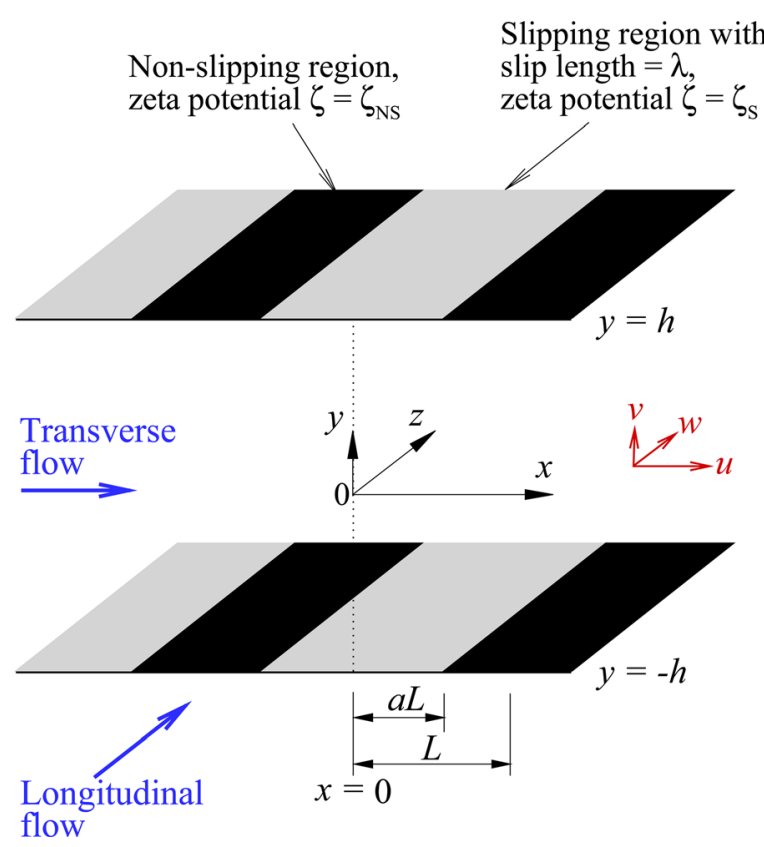

FIG. 1. (Color online) Electrokinetic longitudinal or transverse flow through a parallel-plate channel with walls which are patterned with a periodic array of stripes. One unit of wall pattern consists of a non-slipping stripe of zeta potential $\zeta_{\mathrm{NS}}$, and a slipping stripe of slip length $\lambda$ and zeta potential $\zeta_{\mathrm{S}}$. The two walls are arranged in-phase with each other so that the flow is symmetrical about the centerline of the channel. The $x$ - and $z$-axes are respectively normal and parallel to the stripes, while the $y$-axis is perpendicular to the channel walls. The $x$-, $y$-, and $z$-velocity components are denoted by $(u, v, w)$. Longitudinal flow is unidirectional flow purely along the $z$-direction, and transverse flow is two-dimensional flow in the $(x, y)$ plane. where $\nabla \equiv(\partial / \partial x, \partial / \partial y)$ and $\kappa$ is the inverse of the Debye length (a measure of the thickness of the electric double layer) given by

$$
\kappa=\left(\frac{2 z_{0}^{2} e^{2} n_{0}}{\epsilon k_{B} T}\right)^{1 / 2},
$$

where for a $\left(z_{0}: z_{0}\right)$ symmetrical electrolyte, $z_{0}$ is the valence of the electrolyte, $e$ is the elementary charge, $n_{0}$ is the bulk concentration of the ions at the neutral state, $\epsilon$ is the dielectric constant of the fluid, $k_{B}$ is the Boltzmann constant, and $T$ is the absolute temperature. The Debye-Hückel approximation is valid for $z_{0} e \psi / k_{B} T \ll 1$.

For the present problem, $\psi$ is even in both $x$ and $y$. The solution to Eq. (1) is expressible by

$$
\psi(x, y)=A_{0} \frac{\cosh (\kappa y)}{\cosh (\kappa h)}+\sum_{n=1}^{\infty} A_{n} \cos \left(\alpha_{n} x\right) \frac{\cosh \left(\beta_{n} y\right)}{\cosh \left(\beta_{n} h\right)},
$$

where $\alpha_{n}=n \pi / L$ and $\beta_{n}^{2}=\alpha_{n}^{2}+\kappa^{2}$. The coefficients $A_{0,1,2 .}$ are to be determined on applying the boundary condition

$$
\psi(x, h)=A_{0}+\sum_{n=1}^{\infty} A_{n} \cos \left(\alpha_{n} x\right)=\left\{\begin{array}{ll}
\zeta_{\mathrm{S}} & \text { in } 0<x<a L \\
\zeta_{\mathrm{NS}} & \text { in } a L<x<L
\end{array} .\right.
$$

For this square-wave pattern, the Fourier series coefficients are readily found to be

$$
A_{0}=a \zeta_{\mathrm{S}}+(1-a) \zeta_{\mathrm{NS}}, \quad A_{n}=2\left(\zeta_{\mathrm{S}}-\zeta_{\mathrm{NS}}\right) \frac{\sin \left(\alpha_{n} a L\right)}{\alpha_{n} L} .
$$

The charge density $\rho_{e}(x, y)$ can then be found from the Poisson equation

$$
\rho_{e}=-\epsilon \nabla^{2} \psi=-\epsilon \kappa^{2} \psi .
$$

The electric potential $\psi(x, y)$ described above is the potential due to the electric double layer at the equilibrium state. In the presence of an externally applied electric field $\vec{E}_{\text {ext }}=\left(E_{x}, 0, E_{z}\right)$, where $E_{x}$ and $E_{z}$ are constants, the total potential is

$$
\psi_{\text {tot }}(x, y, z)=\psi(x, y)-(x, y, z) \cdot \vec{E}_{\mathrm{ext}} .
$$

Owing to the free charges in the fluid, a Lorentz body force term $\rho_{e} \vec{E}$ is added to the momentum equation, where $\rho_{e}$ is the charge density given by Eq. (6), and

$$
\vec{E}=-\left(\frac{\partial}{\partial x}, \frac{\partial}{\partial y}, \frac{\partial}{\partial z}\right) \psi_{\mathrm{tot}}=-\left(\frac{\partial}{\partial x}, \frac{\partial}{\partial y}, 0\right) \psi+\vec{E}_{\mathrm{ext}}
$$

is the total electric field strength. Let us consider flow driven by pressure gradient and electric field which are in a direction either parallel or normal to the stripes. We consider only low-Reynolds-number flows so that the inertia can be ignored in the following analysis. We shall work only in the low-Dukhin limit, ${ }^{16}$ where the applied electric and flow fields do not disturb the EDL significantly from its equilibrium state. The Stokes equations are solved by the method of 
eigenfunction expansion where the coefficients are determined by point-matching the mixed boundary conditions. Similar solution techniques have been applied by $\mathrm{Ng}$ and coauthors ${ }^{17-20}$ to Stokes flow through channels with superhydrophobic surfaces made up of alternating slipping stripes.

Before we proceed, let us introduce the following normalization:

$$
\left.\begin{array}{l}
(\hat{x}, \hat{y}, \hat{h}, \hat{\lambda})=(x, y, h, \lambda) / L, \quad\left(\hat{\alpha}_{n}, \hat{\beta}_{n}, \hat{\kappa}\right)=\left(\alpha_{n}, \beta_{n}, \kappa\right) L, \\
\left(\hat{A}_{0}, \hat{A}_{n}, \hat{\zeta}_{\mathrm{S}}\right)=\left(A_{0}, A_{n}, \zeta_{\mathrm{S}}\right) / \zeta_{\mathrm{NS}},
\end{array}\right\}
$$

by which

$$
\hat{A}_{0}=a \hat{\zeta}_{\mathrm{S}}+1-a, \quad \hat{A}_{n}=2\left(\hat{\zeta}_{\mathrm{S}}-1\right) \frac{\sin \left(\hat{\alpha}_{n} a\right)}{\hat{\alpha}_{n}}, \quad \hat{\alpha}_{n}=n \pi .
$$

Equation (4) becomes

$$
\hat{A}_{0}+\sum_{n=1}^{\infty} \hat{A}_{n} \cos \left(\hat{\alpha}_{n} \hat{x}\right)=\left\{\begin{array}{cc}
\hat{\zeta}_{\mathrm{S}} & \text { in } 0<\hat{x}<a \\
1 & \text { in } a<\hat{x}<1
\end{array},\right.
$$

which will be used in our later derivations.

\section{A. Longitudinal flow}

For flow driven by a constant pressure gradient $P_{z}=-d p / d z$ and a constant electric field strength $\vec{E}_{\text {ext }}=\left(0,0, E_{z}\right)$ both purely in the $z$-direction (i.e., parallel to the stripes), the flow is unidirectional along the $z$-direction, and the velocity $w(x, y)$ is governed by

$$
0=-P_{z}+\mu \nabla^{2} w+\rho_{e} E_{z},
$$

where $\mu$ is the dynamic viscosity of the fluid. By linearity, we may decompose the velocity into components due solely to either the pressure or the electric forcing: $w=w_{\mathrm{PO}}+w_{\mathrm{EO}}$. By convention, the two types of flow are termed Poiseuille (PO) flow and electroosmotic (EO) flow, respectively. The two forcings give rise to the following velocity components, which are even in both $x$ and $y$

$$
\begin{aligned}
w_{\mathrm{PO}}(\hat{x}, \hat{y})= & \left\{\frac{\hat{h}^{2}}{2}\left(1-\frac{\hat{y}^{2}}{\hat{h}^{2}}\right)+\hat{h} \hat{\delta}_{\|}\right. \\
& \left.+\hat{h} \sum_{n=1}^{\infty} \hat{C}_{n} \cos \left(\hat{\alpha}_{n} \hat{x}\right) \frac{\cosh \left(\hat{\alpha}_{n} \hat{y}\right)}{\cosh \left(\hat{\alpha}_{n} \hat{h}\right)}\right\} P_{z}^{*}, \\
w_{\mathrm{EO}}(\hat{x}, \hat{y})=- & \left\{\hat{A}_{0} \frac{\cosh (\hat{\kappa} \hat{y})}{\cosh (\hat{\kappa} \hat{h})}+\hat{B}_{0}\right. \\
+ & \left.\sum_{n=1}^{\infty} \cos \left(\hat{\alpha}_{n} \hat{x}\right)\left[\hat{A}_{n} \frac{\cosh \left(\hat{\beta}_{n} \hat{y}\right)}{\cosh \left(\hat{\beta}_{n} \hat{h}\right)}+\hat{B}_{n} \frac{\cosh \left(\hat{\alpha}_{n} \hat{y}\right)}{\cosh \left(\hat{\alpha}_{n} \hat{h}\right)}\right]\right\} E_{z}^{*},
\end{aligned}
$$

where

$$
P_{z}^{*} \equiv-\frac{L^{2}}{\mu} P_{z} \quad \text { and } \quad E_{z}^{*} \equiv-\frac{\epsilon \zeta_{\mathrm{NS}}}{\mu} E_{z}
$$

are the forcing parameters with dimensions of velocity, and the hatted terms are dimensionless quantities defined in Eq. (9). Note that we have expressed the results in such a way that the forcings have the same dimensions as the response, while the phenomenological coefficients are all non-dimensional.

The parameter $\hat{\delta}_{\|}$in Eq. (13) is the dimensionless (normalized by $L$ ) effective or macroscopic hydrodynamic slip length for flow parallel to the stripes. The coefficients $\hat{\delta}_{\|}, \hat{C}_{n}$, $\hat{B}_{0}$, and $\hat{B}_{n}$ are to be determined on applying the mixed-type boundary condition

$$
w= \begin{cases}-\hat{\lambda} \partial w / \partial \hat{y} & \text { in } 0<\hat{x}<a, \hat{y}=\hat{h} \\ 0 & \text { in } a<\hat{x}<1, \hat{y}=\hat{h}\end{cases}
$$

where $\hat{\lambda}$ is the dimensionless microscopic slip length of the slipping stripes. Such a heterogeneous Navier's slip boundary condition has been used previously by, among others, Squires, ${ }^{9}$ Bahga et al.,${ }^{12}$ Zhao, ${ }^{14}$ and Belyaev and Vinogradova $^{15}$ for EO flow over a superhydrophobic surface. On substituting the velocities in Eqs. (13) and (14) into the condition above, the following equations are obtained:

$$
\begin{gathered}
\sum_{n=1}^{M}\left[\cos \left(\hat{\alpha}_{n} \hat{x}\right)-\cos \left(\hat{\alpha}_{n}\right)+\hat{\alpha}_{n} \hat{\lambda} \cos \left(\hat{\alpha}_{n} \hat{x}\right) \tanh \left(\hat{\alpha}_{n} \hat{h}\right)\right] \hat{C}_{n} \\
=\hat{\lambda} \quad(0<\hat{x}<a), \\
\sum_{n=1}^{M}\left[\cos \left(\hat{\alpha}_{n} \hat{x}\right)-\cos \left(\hat{\alpha}_{n}\right)\right] \hat{C}_{n}=0 \quad(a<\hat{x}<1), \\
\hat{\delta}_{\|}=-\sum_{n=1}^{M} \cos \left(\hat{\alpha}_{n}\right) \hat{C}_{n}, \\
\sum_{n=1}^{M}\left[\cos \left(\hat{\alpha}_{n} \hat{x}\right)-\cos \left(\hat{\alpha}_{n}\right)+\hat{\alpha}_{n} \hat{\lambda} \cos \left(\hat{\alpha}_{n} \hat{x}\right) \tanh \left(\hat{\alpha}_{n} \hat{h}\right)\right] \hat{B}_{n} \\
=-\hat{A}_{0} \hat{\kappa} \hat{\lambda} \tanh (\hat{\kappa} \hat{h})-\sum_{n=1}^{M} \hat{A}_{n}\left[\cos \left(\hat{\alpha}_{n} \hat{x}\right)-\cos \left(\hat{\alpha}_{n}\right)\right. \\
\left.+\hat{\beta}_{n} \hat{\lambda} \cos \left(\hat{\alpha}_{n} \hat{x}\right) \tanh \left(\hat{\beta}_{n} \hat{h}\right)\right] \quad(0<\hat{x}<a), \\
\sum_{n=1}^{M}\left[\cos \left(\hat{\alpha}_{n} \hat{x}\right)-\cos \left(\hat{\alpha}_{n}\right)\right] \hat{B}_{n}=0 \quad(a<\hat{x}<1), \\
\hat{B}_{0}=-1-\sum_{n=1}^{M} \cos \left(\hat{\alpha}_{n}\right) \hat{B}_{n},
\end{gathered}
$$

where we have truncated the series each to $M$ terms. In the sense of point collocation, we impose the two pairs of Eqs. (17) and (18) and Eqs. (20) and (21) at $M$ discrete points evenly distributed in the domain $0 \leq \hat{x}<1$ while avoiding the junction point $\hat{x}=a$. This forms two $M \times M$ systems of linear equations for the unknowns $\hat{C}_{1, \cdots, M}$ and $\hat{B}_{1, \cdots, M}$, which can be solved readily by a standard routine. The coefficients $\hat{\delta}_{\|}$and $\hat{B}_{0}$ are then found from Eqs. (19) and (22).

Taking average over a periodic unit of cross-sectional area, we get the mean velocity for the longitudinal flow 


$$
\bar{w}=L_{11}^{\|} P_{z}^{*}+L_{12}^{\|} E_{z}^{*},
$$

where

$$
\begin{gathered}
L_{11}^{\|}=\hat{h}^{2}\left(\frac{1}{3}+\frac{\hat{\delta}}{\hat{h}}\right), \\
L_{12}^{\|}=-\hat{A}_{0} \frac{\tanh (\hat{\kappa} \hat{h})}{\hat{\kappa} \hat{h}}-\hat{B}_{0}
\end{gathered}
$$

are dimensionless phenomenological coefficients known as the hydrodynamic conductance and the streaming flow conductance, respectively. The driving forces $P_{z}^{*}$ and $E_{z}^{*}$ are defined in Eq. (15), where $E_{z}^{*}$ is also known as the HelmholtzSmoluchowski velocity.

The electric current density arising from conduction and convection is given by

$$
I_{z}=\sigma E_{z}+\rho_{e} w,
$$

where $\sigma$ is the electric conductivity of the fluid. The electric conductivity is a function of the charge density and therefore a function of space. Its variation is, however, small under the condition of low potentials. ${ }^{4}$ Here, for simplicity, we assume that $\sigma$ is a constant. On substituting Eqs. (3), (13), and (14) for $\rho_{e}=-\epsilon \kappa^{2} \psi$ and $w=w_{\mathrm{PO}}+w_{\mathrm{EO}}$ and taking average over a unit sectional area, we get the following expression for the mean current density:

$$
\bar{I}_{z}=L_{21}^{\|} P_{z}^{* *}+L_{22}^{\|} E_{z}^{* *}
$$

where

$$
P_{z}^{* *} \equiv \frac{\epsilon \zeta_{\mathrm{NS}}}{\mu} P_{z} \quad \text { and } \quad E_{z}^{* *} \equiv \frac{\epsilon^{2} \zeta_{\mathrm{NS}}^{2}}{\mu L^{2}} E_{z}
$$

are the forcing parameters with dimensions of current density. Note that they are so defined that their ratio is equal to that of those with dimensions of velocity: $E_{z}^{* *} / P_{z}^{* *}=E_{z}^{*} / P_{z}^{*}$. The dimensionless phenomenological coefficients are given by

$$
\begin{aligned}
& L_{21}^{\|}= \hat{A}_{0}\left[\left(\hat{\kappa} \hat{\delta}_{\|}-\frac{1}{\hat{\kappa} \hat{h}}\right) \tanh (\hat{\kappa} \hat{h})+1\right] \\
&+\frac{1}{2} \sum_{n=1}^{M} \hat{A}_{n} \hat{C}_{n}\left[\hat{\beta}_{n} \tanh \left(\hat{\beta}_{n} \hat{h}\right)-\hat{\alpha}_{n} \tanh \left(\hat{\alpha}_{n} \hat{h}\right)\right], \quad \text { (29) } \\
& L_{22}^{\|}=\hat{\kappa}^{2} \hat{\sigma}-\frac{\hat{\kappa}^{2} \hat{A}_{0}^{2}}{2}\left[\operatorname{sech}(\hat{\kappa} \hat{h})+\frac{\tanh (\hat{\kappa} \hat{h})}{\hat{\kappa} \hat{h}}\right] \\
&-\frac{\hat{\kappa} \hat{A}_{0} \hat{B}_{0}}{\hat{h}} \tanh (\hat{\kappa} \hat{h})-\frac{\hat{\kappa}^{2}}{4} \sum_{n=1}^{M} \hat{A}_{n}^{2}\left[\operatorname{sech}^{2}\left(\hat{\beta}_{n} \hat{h}\right)+\frac{\tanh \left(\hat{\beta}_{n} \hat{h}\right)}{\hat{\beta}_{n} \hat{h}}\right] \\
&-\frac{1}{2 \hat{h}} \sum_{n=1}^{M} \hat{A}_{n} \hat{B}_{n}\left[\hat{\beta}_{n} \tanh \left(\hat{\beta}_{n} \hat{h}\right)-\hat{\alpha}_{n} \tanh \left(\hat{\alpha}_{n} \hat{h}\right)\right], \quad(30)
\end{aligned}
$$

where

$$
\hat{\sigma}=\frac{\mu \sigma}{\epsilon^{2} \kappa^{2} \zeta_{\mathrm{NS}}^{2}}
$$

is the normalized electric conductivity. The coefficients $L_{21}^{\|}$ and $L_{22}^{\|}$are respectively known as the streaming current conductance and the electrical conductance. Equations (23) and (27) are the Onsager relations for the flow and ionic fluxes under longitudinal forcings. By reciprocity of the Onsager relations, the non-conjugate coefficients are equal to each other: $L_{12}^{\|}=L_{21}^{\|}$. A simple yet formal proof of this symmetry is as follows. When normalized with respect to their respective forcings, the two velocity components are governed by, from Eq. (12),

$$
\begin{gathered}
\nabla^{2} w_{\mathrm{EO}}+\rho_{e}=0 \\
\nabla^{2} w_{\mathrm{PO}}+1=0
\end{gathered}
$$

On multiplying Eq. (32) by $w_{\text {PO }}$, we get

$$
\begin{gathered}
w_{\mathrm{PO}} \nabla^{2} w_{\mathrm{EO}}+\rho_{e} w_{\mathrm{PO}}=0 \\
\Rightarrow \nabla \cdot\left(w_{\mathrm{PO}} \nabla w_{\mathrm{EO}}-w_{\mathrm{EO}} \nabla w_{\mathrm{PO}}\right)+w_{\mathrm{EO}} \nabla^{2} w_{\mathrm{PO}}+\rho_{e} w_{\mathrm{PO}}=0 \\
\Rightarrow \nabla \cdot\left(w_{\mathrm{PO}} \nabla w_{\mathrm{EO}}-w_{\mathrm{EO}} \nabla w_{\mathrm{PO}}\right)-w_{\mathrm{EO}}+\rho_{e} w_{\mathrm{PO}}=0,
\end{gathered}
$$

where Eq. (33) is used in the last step. On integrating over a unit sectional area, one finds that the integral of the divergence term above will vanish. This is because, using Gauss's law,

$$
\begin{aligned}
& \iint_{\Omega} \nabla \cdot\left(w_{\mathrm{PO}} \nabla w_{\mathrm{EO}}-w_{\mathrm{EO}} \nabla w_{\mathrm{PO}}\right) \mathrm{d} A \\
& =\int_{\partial \Omega}\left(w_{\mathrm{PO}} \nabla w_{\mathrm{EO}}-w_{\mathrm{EO}} \nabla w_{\mathrm{PO}}\right) \cdot \vec{n} \mathrm{~d} S,
\end{aligned}
$$

where $\vec{n}$ is the unit outward normal to the boundary $\partial \Omega$ of the unit sectional area $\Omega$. Over the boundary where the condition is no slip, $w_{\mathrm{PO}}=w_{\mathrm{EO}}=0$, the integrand of the line integral is zero. Over the boundary where the condition is partial slip, $w_{\mathrm{PO}}=-\lambda \nabla w_{\mathrm{PO}} \cdot \vec{n}$ and $w_{\mathrm{EO}}=-\lambda \nabla w_{\mathrm{EO}} \cdot \vec{n}$, the integrand is also zero. Over the remaining boundary which is periodic, the line integral will cancel to zero. Hence, the sectional averaging of Eq. (34) will result in

$$
\bar{w}_{\mathrm{EO}}=\overline{\rho_{e} w_{\mathrm{PO}}},
$$

which serves as a proof to the equality $L_{12}^{\|}=L_{21}^{\|}$. We have confirmed with numerical calculations that Eqs. (25) and (29) will indeed produce the same values for the same inputs.

Let us look into two cases in particular. First, consider the case when the wall potential is uniform so that $\zeta_{\mathrm{NS}}=\zeta_{\mathrm{S}}$ or $\hat{\zeta}_{\mathrm{S}}=1$. It follows that $\hat{A}_{0}=1$ and $\hat{A}_{n}=0$. It is immediately clear from Eqs. (17), (18), (20), and (21) that $\hat{B}_{n}$ and $\hat{C}_{n}$ are collinear vectors related by $\hat{B}_{n}=-\hat{\kappa} \tanh (\hat{\kappa} \hat{h}) \hat{C}_{n}$. Further using Eqs. (19) and (22), we get $\hat{B}_{0}=-\hat{\kappa} \hat{\delta}_{\|} \tanh (\hat{\kappa} \hat{h})-1$. Substituting this into Eq. (25), we have the streaming flow conductance be given by

$$
L_{12}^{\|}=1+\hat{\kappa} \hat{\delta}_{\|} \tanh (\hat{\kappa} \hat{h})-\frac{\tanh (\hat{\kappa} \hat{h})}{\hat{\kappa} \hat{h}} \text { for } \quad \hat{\zeta}_{\mathrm{S}}=1 \text {. }
$$


In terms of dimensional quantities, the mean EOF velocity is given by

$$
\begin{aligned}
\bar{w}_{\mathrm{EO}} & =\left[1+\kappa \delta_{\|} \tanh (\kappa h)-\frac{\tanh (\kappa h)}{\kappa h}\right]\left(-\frac{\epsilon \zeta}{\mu} E_{z}\right) \text { for } \\
\zeta_{\mathrm{NS}} & =\zeta_{\mathrm{S}}=\zeta .
\end{aligned}
$$

Note that the EO flow is enhanced by the slip through the term $\kappa \delta_{\|} \tanh (\kappa h)$, which is linearly proportional to the effective slip length $\delta_{\|}$. A limiting case of this result has been derived previously by Squires, ${ }^{9}$ whose formula, valid for a thin EDL, reads as follows:

$$
\mathbf{U}^{\mathrm{EOF}}=-\frac{\epsilon \zeta \mathbf{E}}{\mu} \cdot\left(\mathbf{I}+\kappa \mathbf{b}_{E}\right) \text { for uniform zeta potential } \zeta
$$

where $\mathbf{U}^{\mathrm{EOF}}$ is the effective EO flow velocity over a surface with an arbitrary distribution of microscopic slip, $\mathbf{E}$ is the electric field vector, $\mathbf{I}$ is the identity tensor, and $\mathbf{b}_{E}$ is the effective slip length tensor. This result led Squires to remark that EOF with a constant wall potential over an arbitrarily slipping surface is enhanced by precisely the same amount as would be found by naively assuming the effective slip length to apply homogeneously. We have here obtained a more general expression, and Squires' remark still holds. The effective slip length that has been found in the hydrodynamic problem can be used directly in the electrokinetic problem as if the slip were homogeneous (i.e., with a constant microscopic slip length equal to the macroscopic effective slip length), as long as the wall potential is constant. Squires ${ }^{9}$ made the following assumptions in his proof: a very thick channel $\hat{h} \gg 1$, and an extremely thin electric double layer $\hat{\kappa} \gg 1$. Equation (38) is a more general result as it holds even for finite values of $\hat{h}$ and $\hat{\kappa}$ (i.e., arbitrary channel height and EDL thickness as long as the EDLs are not strongly overlapped). For longitudinal flow through a thick channel with perfect slip stripes, the formula by Philip ${ }^{21}$ can be used

$$
\delta_{\|}=\frac{2 L}{\pi} \ln \left[\sec \left(\frac{\pi a}{2}\right)\right] \text { for } \quad \hat{\lambda}=\infty, \hat{h} \gg 1 .
$$

When the stripes are partial slipping, one may use the approximate formula derived by Belyaev and Vinogradova ${ }^{22}$

$$
\delta_{\|} \simeq \frac{\frac{2 L}{\pi} \ln \left[\sec \left(\frac{\pi a}{2}\right)\right]}{1+\frac{2 L}{\pi \lambda} \ln \left[\sec \left(\frac{\pi a}{2}\right)+\tan \left(\frac{\pi a}{2}\right)\right]} \quad \text { for any } \hat{\lambda}, \text { and } \hat{h} \gg 1
$$

In this work, finite channel height is considered, and the effective slip length $\hat{\delta}_{\|}$is to be computed numerically as mentioned above. Further, for a very thin electric double layer, the EOF velocity in Eq. (38) reduces to

$$
\bar{w}_{\mathrm{EO}} \approx\left(1+\kappa \delta_{\|}\right)\left(-\frac{\epsilon \zeta}{\mu} E_{z}\right) \text { for } \kappa h \gg 1,
$$

which matches the longitudinal component of Eq. (39) derived by Squires. ${ }^{9}$ Hence, in this thin EDL limit, the EOF is enhanced by a factor equal to the ratio of the effective slip length to the Debye length. Such a linear factor of EOF enhancement due to boundary slip was first proposed by Churaev et al., ${ }^{6}$ and later verified with molecular dynamics simulations by Joly et al. ${ }^{23}$ By this factor, slip lengths in the nano- to micrometer range can result in a very large enhancement, as much as two orders of magnitude, owing to the much thinner electric double layer. ${ }^{24}$ Such an enhancement was measured by Bouzigues et al. ${ }^{25}$ Any measurement of the zeta potential in the presence of slip will lead to an apparent zeta potential that is larger than the true one by the enhancement factor $1+\kappa \delta$. We caution that one should be mindful of the sensitivity of this result near the limit $a=1$. We shall show in Sec. III, with numerical results, how the slip-induced EOF enhancement will vary as a function of the area function $a$.

Second, let us consider, for any values of the wall potentials, the limiting condition of a very thin EDL, $\hat{\kappa} \gg 1$, such that $\hat{\kappa} \hat{h} \gg 1$ and $\hat{\beta}_{n} \approx \hat{\kappa}$. From Eq. (11), we get $\sum_{n=1}^{M} \hat{A}_{n} \cos \left(\hat{\alpha}_{n} \hat{x}\right)=\hat{\zeta}_{\mathrm{S}}-\hat{A}_{0} \quad$ where $0<\hat{x}<a$, and $\sum_{n=1}^{M} \hat{A}_{n} \cos \left(\hat{\alpha}_{n}\right)=1-\hat{A}_{0}$. Under this limiting condition, the right-hand side of Eq. (20) can be simplified to

$$
\begin{aligned}
\operatorname{RHS}(20)= & -\hat{A_{0}} \hat{\kappa} \hat{\lambda} \tanh (\hat{\kappa} \hat{h})-\sum_{n=1}^{M} \hat{A}_{n} \cos \left(\hat{\alpha}_{n} \hat{x}\right) \\
& +\sum_{n=1}^{M} \hat{A}_{n} \cos \left(\hat{\alpha}_{n}\right) \\
& -\sum_{n=1}^{M} \hat{A}_{n} \hat{\beta}_{n} \hat{\lambda} \cos \left(\hat{\alpha}_{n} \hat{x}\right) \tanh \left(\hat{\beta}_{n} \hat{h}\right) \quad(0<\hat{x}<a) \\
& \approx 1-\hat{\zeta}_{\mathrm{S}}(1+\hat{\kappa} \hat{\lambda}) \quad \text { when } \hat{\kappa} \gg 1 .
\end{aligned}
$$

Since this right-hand side has become a constant independent of $\hat{x}$, then from Eqs. (17), (18), (20), and (21) we see that $\hat{B}_{n}$ and $\hat{C}_{n}$ are nearly collinear vectors, related by $\hat{B}_{n} \approx\left[1-\hat{\zeta}_{\mathrm{S}}(1+\hat{\kappa} \hat{\lambda})\right] \hat{C}_{n} / \hat{\lambda}$. It follows from Eqs. (19), (22), and (25) that

$$
L_{12}^{\|} \approx-\hat{B}_{0} \approx 1+\frac{\hat{\delta}_{\|}}{\hat{\lambda}}\left[\hat{\zeta}_{\mathrm{S}}(1+\hat{\kappa} \hat{\lambda})-1\right] \text { for } \hat{\kappa} \gg 1,
$$

which agrees with the expression for EO mobility recently obtained by Belyaev and Vinogradova, ${ }^{15}$ who considered EO flow over a surface in a thick channel with stripes of partial slip. Here, we have shown that the same expression holds even for a channel of finite height, where the channel height will affect the effective slip length $\hat{\delta}_{\|}$.

Some particular cases readily follow from Eq. (44). For equal wall potentials $\hat{\zeta}_{\mathrm{S}}=1$, we get $L_{12}^{\|} \approx 1+\hat{\kappa} \hat{\delta}_{\|}$, which recovers Eq. (42). For perfect-slip stripes $\hat{\lambda}=\infty$, we get $L_{12}^{\|} \approx 1+\hat{\zeta}_{\mathrm{S}} \hat{\kappa} \hat{\delta}_{\|}$as has been previously deduced by Squires ${ }^{9}$ and Bahga et al. ${ }^{12}$ (see also Vinogradova and Belyaev, ${ }^{26}$ who have corrected some erroneous expressions given in Bahga et $a l .{ }^{12}$ ). If the perfect-slipping region is uncharged, $\hat{\zeta}_{\mathrm{S}}=0$, the result further reduces to $L_{12}^{\|} \approx 1$. In other words, 
in this limiting case, the EOF velocity is simply equal to the Helmholtz-Smoluchowski velocity without seeing any slip effect on it

$$
\bar{w}_{\mathrm{EO}}=-\frac{\epsilon \zeta_{\mathrm{NS}}}{\mu} E_{z} \quad \text { for } \quad \hat{\zeta}_{\mathrm{S}}=0, \quad \hat{\lambda}=\infty, \quad \hat{\kappa} \gg 1
$$

This interesting result was first pointed out by Squires, ${ }^{9}$ whose formula, valid for a thin EDL, reads as follows:

$$
\mathbf{U}^{\mathrm{EOF}}=-\frac{\epsilon \zeta_{\mathrm{NS}} \mathbf{E}}{\mu} \quad \text { for uncharged slipping regions } \zeta_{\mathrm{S}}=0
$$

This result led Squires to remark that surfaces with uncharged perfect-slipping regions will give rise to no enhancement due to the slip, instead giving precisely the same EOF as if the surface were non-slipping and homogeneously charged. This phenomenon, confirmed by molecular dynamics simulations by Huang et al., ${ }^{27}$ can be reasoned easily. Under the condition $\kappa h \gg 1$, the EOF is a plug flow with the velocity equal to the HelmholtzSmoluchowski velocity. The plug flow, which itself is unsheared, will experience neither retardation nor forcing from the perfect-slip and uncharged regions of the wall. As a result, the hydrodynamic slip loses influence as the flow already attains perfect EO slip. We caution that the limiting results in Eqs. (44) and (45) are valid only for very large $\hat{\kappa}$. The range of validity of these limiting relations will be examined in Sec. III.

\section{B. Transverse flow}

We next consider flow that is driven by a constant pressure gradient $P_{x}=-d p / d x$ and a constant electric field strength $\vec{E}_{\text {ext }}=\left(E_{x}, 0,0\right)$ both purely in the $x$-direction (i.e., perpendicular to the stripes), the flow is two-dimensional in the $(x, y)$ plane, and the velocity $\vec{u} \equiv(u(x, y), v(x, y))$ is governed by

$$
\begin{gathered}
\nabla \cdot \vec{u}=0, \\
0=-\left(P_{x}, 0\right)-\nabla p+\mu \nabla^{2} \vec{u}+\rho_{e} \vec{E},
\end{gathered}
$$

where $p(x, y)$ is the locally induced pressure, and the electric field strength is $\vec{E}=-\nabla \psi+\left(E_{x}, 0\right)$ in which $\psi$ is the electric double-layer potential given in Eq. (3). The primitive variables $(u, v, p)$ are periodic functions of $x$ as a result of the periodic stick-slip boundary conditions. By symmetry or anti-symmetry of the flow field, $u$ is even in both $x$ and $y, v$ is odd in both $x$ and $y$, and $p$ is odd in $x$ but even in $y$.

Let us decompose the flow into the pressure-driven or Poiseuille (PO) and the electro-osmotic (EO) components: $\vec{u}=\vec{u}_{\mathrm{PO}}+\vec{u}_{\mathrm{EO}}$. The corresponding momentum equations are

$$
0=-\left(P_{x}, 0\right)-\nabla p_{\mathrm{PO}}+\mu \nabla^{2} \vec{u}_{\mathrm{PO}},
$$

and

$$
0=-\nabla p_{\mathrm{EO}}+\mu \nabla^{2} \vec{u}_{\mathrm{EO}}+\rho_{e} \vec{E} .
$$

The hydrodynamic velocity components $\vec{u}_{\mathrm{PO}}=\left(u_{\mathrm{PO}}, \nu_{\mathrm{PO}}\right)$ are readily found to be

$$
\begin{gathered}
u_{\mathrm{PO}}(\hat{x}, \hat{y})=\left\{\frac{\hat{h}^{2}}{2}\left(1-\frac{\hat{y}^{2}}{\hat{h}^{2}}\right)+\hat{h} \hat{\delta}_{\perp}+\hat{h} \sum_{n=1}^{\infty} \hat{D}_{n} \frac{\cos \left(\hat{\alpha}_{n} \hat{x}\right)}{\cosh \left(\hat{\alpha}_{n} \hat{h}\right)} \times\left[\cosh \left(\hat{\alpha}_{n} \hat{y}\right)-\frac{\tanh \left(\hat{\alpha}_{n} \hat{h}\right)}{\hat{\alpha}_{n} \hat{h}}\left[\cosh \left(\hat{\alpha}_{n} \hat{y}\right)+\hat{\alpha}_{n} \hat{y} \sinh \left(\hat{\alpha}_{n} \hat{y}\right)\right]\right]\right\} P_{x}^{*}, \\
v_{\mathrm{PO}}(\hat{x}, \hat{y})=\left\{\hat{h} \sum_{n=1}^{\infty} \hat{D}_{n} \frac{\sin \left(\hat{\alpha}_{n} \hat{x}\right)}{\cosh \left(\hat{\alpha}_{n} \hat{h}\right)} \times\left[\sinh \left(\hat{\alpha}_{n} \hat{y}\right)-\tanh \left(\hat{\alpha}_{n} \hat{h}\right)\left(\frac{\hat{y}}{\hat{h}}\right) \cosh \left(\hat{\alpha}_{n} \hat{y}\right)\right]\right\} P_{x}^{*},
\end{gathered}
$$

where $\hat{\delta}_{\perp}$ is the dimensionless effective hydrodynamic slip length for flow perpendicular to the stripes on the wall and $P_{x}^{*} \equiv-\left(L^{2} / \mu\right) P_{x}$ is the pressure forcing parameter with dimensions of velocity.

For the EOF, after substituting for $\rho_{e}$ and $\vec{E}$, Eq. (50) can be written as

$$
0=-\nabla p_{\mathrm{EO}}^{\prime}+\mu \nabla^{2} \vec{u}_{\mathrm{EO}}-\epsilon \kappa^{2} \psi\left(E_{x}, 0\right)
$$

where $p_{\mathrm{EO}}^{\prime}=p_{\mathrm{EO}}-\epsilon \kappa^{2} \psi^{2} / 2$ is the effective pressure (i.e., electrostatic pressure being subtracted from the total pressure) of the EOF. After some algebra solving for the particular solution, the velocity components $\vec{u}_{\mathrm{EO}}=\left(u_{\mathrm{EO}}, v_{\mathrm{EO}}\right)$ satisfying continuity and zero normal flux on the walls are found to be

$$
\begin{aligned}
& u_{\mathrm{EO}}(\hat{x}, \hat{y})=-\left\{\hat{A}_{0} \frac{\cosh (\hat{\kappa} \hat{y})}{\cosh (\hat{\kappa} \hat{h})}+\hat{F}_{0}+\sum_{n=1}^{\infty} \hat{A}_{n} \frac{\cos \left(\hat{\alpha}_{n} \hat{x}\right)}{\left(1-\hat{\alpha}_{n}^{2} / \hat{\beta}_{n}^{2}\right)}\left[\frac{\cosh \left(\hat{\beta}_{n} \hat{y}\right)}{\cosh \left(\hat{\beta}_{n} \hat{h}\right)}-\frac{\hat{\alpha}_{n}}{\hat{\beta}_{n}} \frac{\tanh \left(\hat{\beta}_{n} \hat{h}\right)}{\cosh \left(\hat{\alpha}_{n} \hat{h}\right)} \times\left(\frac{\cosh \left(\hat{\alpha}_{n} \hat{y}\right)}{\hat{\alpha}_{n} \hat{h}}+\frac{\hat{y}}{\hat{h}} \sinh \left(\hat{\alpha}_{n} \hat{y}\right)\right)\right]\right. \\
& \left.+\sum_{n=1}^{\infty} \hat{F}_{n} \frac{\cos \left(\hat{\alpha}_{n} \hat{x}\right)}{\cosh \left(\hat{\alpha}_{n} \hat{h}\right)}\left[\cosh \left(\hat{\alpha}_{n} \hat{y}\right)-\frac{\tanh \left(\hat{\alpha}_{n} \hat{h}\right)}{\hat{\alpha}_{n} \hat{h}} \times\left[\cosh \left(\hat{\alpha}_{n} \hat{y}\right)+\hat{\alpha}_{n} \hat{y} \sinh \left(\hat{\alpha}_{n} \hat{y}\right)\right]\right]\right\} E_{x}^{*},
\end{aligned}
$$




$$
\begin{aligned}
v_{\mathrm{EO}}(\hat{x}, \hat{y})= & -\left\{\sum_{n=1}^{\infty} \hat{A}_{n} \frac{\hat{\alpha}_{n} \sin \left(\hat{\alpha}_{n} \hat{x}\right)}{\hat{\beta}_{n}\left(1-\hat{\alpha}_{n}^{2} / \hat{\beta}_{n}^{2}\right)}\left[\frac{\sinh \left(\hat{\beta}_{n} \hat{y}\right)}{\cosh \left(\hat{\beta}_{n} \hat{h}\right)}-\frac{\tanh \left(\hat{\beta}_{n} \hat{h}\right)}{\cosh \left(\hat{\alpha}_{n} \hat{h}\right)}\left(\frac{\hat{y}}{\hat{h}}\right) \cosh \left(\hat{\alpha}_{n} \hat{y}\right)\right]\right. \\
& \left.+\sum_{n=1}^{\infty} \hat{F}_{n} \frac{\sin \left(\hat{\alpha}_{n} \hat{x}\right)}{\cosh \left(\hat{\alpha}_{n} \hat{h}\right)}\left[\sinh \left(\hat{\alpha}_{n} \hat{y}\right)-\tanh \left(\hat{\alpha}_{n} \hat{h}\right)\left(\frac{\hat{y}}{\hat{h}}\right) \cosh \left(\hat{\alpha}_{n} \hat{y}\right)\right]\right\} E_{x}^{*},
\end{aligned}
$$

where $\hat{A}_{0}, \hat{A}_{n}$ are given in Eq. (10) and $E_{x}^{*} \equiv-\left(\epsilon \zeta_{\mathrm{NS}} / \mu\right) E_{x}$ is the electric forcing parameter with dimensions of velocity.

Again, we have expressed the results in such a way that the forcings have the same dimensions as the response, while the coefficients are all non-dimensional. In the solutions above, the coefficients $\hat{\delta}_{\perp}, \hat{D}_{n}, \hat{F}_{0}$, and $\hat{F}_{n}$ are to be determined on applying the stick-slip boundary condition

$$
u= \begin{cases}-\hat{\lambda} \partial u / \partial \hat{y} & \text { in } 0<\hat{x}<a, \hat{y}=\hat{h} \\ 0 & \text { in } a<\hat{x}<1, \hat{y}=\hat{h}\end{cases}
$$

On substituting the velocities in Eqs. (51) and (54) into the condition above, the following equations are obtained

$$
\begin{aligned}
& \sum_{n=1}^{M}\left\{\left[\cos \left(\hat{\alpha}_{n} \hat{x}\right)-\cos \left(\hat{\alpha}_{n}\right)\right]\left[\operatorname{sech}^{2}\left(\hat{\alpha}_{n} \hat{h}\right)-\frac{\tanh \left(\hat{\alpha}_{n} \hat{h}\right)}{\hat{\alpha}_{n} \hat{h}}\right]-\left(\frac{2 \hat{\lambda}}{\hat{h}}\right) \cos \left(\hat{\alpha}_{n} \hat{x}\right) \tanh ^{2}\left(\hat{\alpha}_{n} \hat{h}\right)\right\} \hat{D}_{n}=\hat{\lambda} \quad(0<\hat{x}<a) \\
& \sum_{n=1}^{M}\left\{\left[\cos \left(\hat{\alpha}_{n} \hat{x}\right)-\cos \left(\hat{\alpha}_{n}\right)\right]\left[\operatorname{sech}^{2}\left(\hat{\alpha}_{n} \hat{h}\right)-\frac{\tanh \left(\hat{\alpha}_{n} \hat{h}\right)}{\hat{\alpha}_{n} \hat{h}}\right]\right\} \hat{D}_{n}=0 \quad(a<\hat{x}<1), \\
& \hat{\delta}_{\perp}=-\sum_{n=1}^{M} \cos \left(\hat{\alpha}_{n}\right)\left[\operatorname{sech}^{2}\left(\hat{\alpha}_{n} \hat{h}\right)-\frac{\tanh \left(\hat{\alpha}_{n} \hat{h}\right)}{\hat{\alpha}_{n} \hat{h}}\right] \hat{D}_{n} \\
& \sum_{n=1}^{M}\left\{\left[\cos \left(\hat{\alpha}_{n} \hat{x}\right)-\cos \left(\hat{\alpha}_{n}\right)\right]\left[\operatorname{sech}^{2}\left(\hat{\alpha}_{n} \hat{h}\right)-\frac{\tanh \left(\hat{\alpha}_{n} \hat{h}\right)}{\hat{\alpha}_{n} \hat{h}}\right]-\left(\frac{2 \hat{\lambda}}{\hat{h}}\right) \cos \left(\hat{\alpha}_{n} \hat{x}\right) \tanh ^{2}\left(\hat{\alpha}_{n} \hat{h}\right)\right\} \hat{F}_{n} \\
& =-\hat{A_{0}} \hat{\kappa} \hat{\lambda} \tanh (\hat{\kappa} \hat{h})-\sum_{n=1}^{M} \frac{\hat{A}_{n}}{\left(1-\hat{\alpha}_{n}^{2} / \hat{\beta}_{n}^{2}\right)} \times\left\{\left[\cos \left(\hat{\alpha}_{n} \hat{x}\right)-\cos \left(\hat{\alpha}_{n}\right)\right]\left[1-\frac{\tanh \left(\hat{\beta}_{n} \hat{h}\right)}{\hat{\beta}_{n} \hat{h}}-\frac{\hat{\alpha}_{n}}{\hat{\beta}_{n}} \tanh \left(\hat{\beta}_{n} \hat{h}\right) \tanh \left(\hat{\alpha}_{n} \hat{h}\right)\right]\right. \\
& \left.+\hat{\beta}_{n} \hat{\lambda} \cos \left(\hat{\alpha}_{n} \hat{x}\right) \tanh \left(\hat{\beta}_{n} \hat{h}\right)\left[1-2 \frac{\hat{\alpha}_{n}}{\hat{\beta}_{n}} \frac{\tanh \left(\hat{\alpha}_{n} \hat{h}\right)}{\hat{\beta}_{n} \hat{h}}-\frac{\hat{\alpha}_{n}^{2}}{\hat{\beta}_{n}^{2}}\right]\right\} \quad(0<\hat{x}<a), \\
& \sum_{n=1}^{M}\left\{\left[\cos \left(\hat{\alpha}_{n} \hat{x}\right)-\cos \left(\hat{\alpha}_{n}\right)\right]\left[\operatorname{sech}^{2}\left(\hat{\alpha}_{n} \hat{h}\right)-\frac{\tanh \left(\hat{\alpha}_{n} \hat{h}\right)}{\hat{\alpha}_{n} \hat{h}}\right]\right\} \hat{F}_{n}=-\sum_{n=1}^{M} \frac{\hat{A}_{n}}{\left(1-\hat{\alpha}_{n}^{2} / \hat{\beta}_{n}^{2}\right)}\left\{[ \operatorname { c o s } ( \hat { \alpha } _ { n } \hat { x } ) - \operatorname { c o s } ( \hat { \alpha } _ { n } ) ] \left[1-\frac{\tanh \left(\hat{\beta}_{n} \hat{h}\right)}{\hat{\beta}_{n} \hat{h}}\right.\right. \\
& \left.\left.-\frac{\hat{\alpha}_{n}}{\hat{\beta}_{n}} \tanh \left(\hat{\beta}_{n} \hat{h}\right) \tanh \left(\hat{\alpha}_{n} \hat{h}\right)\right]\right\} \quad(a<\hat{x}<1), \\
& \hat{F}_{0}=-\sum_{n=1}^{M} \cos \left(\hat{\alpha}_{n}\right)\left[\operatorname{sech}^{2}\left(\hat{\alpha}_{n} \hat{h}\right)-\frac{\tanh \left(\hat{\alpha}_{n} \hat{h}\right)}{\hat{\alpha}_{n} \hat{h}}\right] \hat{F}_{n}-\hat{A}_{0}-\sum_{n=1}^{M} \frac{\hat{A}_{n} \cos \left(\hat{\alpha}_{n}\right)}{\left(1-\hat{\alpha}_{n}^{2} / \hat{\beta}_{n}^{2}\right)}\left[1-\frac{\tanh \left(\hat{\beta}_{n} \hat{h}\right)}{\hat{\beta}_{n} \hat{h}}-\frac{\hat{\alpha}_{n}}{\hat{\beta}_{n}} \tanh \left(\hat{\beta}_{n} \hat{h}\right) \tanh \left(\hat{\alpha}_{n} \hat{h}\right)\right]
\end{aligned}
$$

where we have truncated the series each to $M$ terms. Again, by the method of point collocation, we impose the two pairs of Eqs. (57) and (58) and Eqs. (60) and (61) at $M$ discrete points evenly distributed in the domain $0 \leq \hat{x}<1$ while avoiding the junction point $\hat{x}=a$. This forms two $M \times M$ sys- tems of linear equations for the unknowns $\hat{D}_{1, \cdots, M}$ and $\hat{F}_{1, \cdots, M}$, which can be solved readily by a standard routine. The coefficients $\hat{\delta}_{\perp}$ and $\hat{F}_{0}$ are then found from Eqs. (59) and (62).

On averaging across the channel height, we get the mean velocity for the transverse flow 


$$
\bar{u}=L_{11}^{\perp} P_{x}^{*}+L_{12}^{\perp} E_{x}^{*},
$$

where

$$
\begin{gathered}
L_{11}^{\perp}=\hat{h}^{2}\left(\frac{1}{3}+\frac{\hat{\delta}_{\perp}}{\hat{h}}\right), \\
L_{12}^{\perp}=-\hat{A}_{0} \frac{\tanh (\hat{\kappa} \hat{h})}{\hat{\kappa} \hat{h}}-\hat{F}_{0}
\end{gathered}
$$

are, respectively, the hydrodynamic conductance and the streaming flow conductance, both dimensionless. The driving forces are $P_{x}^{*} \equiv-\left(L^{2} / \mu\right) P_{x}$ and $E_{x}^{*} \equiv-\left(\epsilon \zeta_{\mathrm{NS}} / \mu\right) E_{x}$, which have dimensions of velocity. The parameter $E_{x}^{*}$ is the Helmholtz-Smoluchowski velocity.

The axial electric current density is given by

$$
I_{x}=\sigma E_{x}+\rho_{e} u,
$$

where $\sigma$ is the electric conductivity of the fluid. On substituting Eqs. (3), (51), and (54) for $\rho_{e}=-\epsilon \kappa^{2} \psi$ and $u=u_{\mathrm{PO}}+u_{\mathrm{EO}}$, and taking average over one periodic unit volume that spans in both streamwise and lateral directions of the channel, we get the following expression for the mean axial current density:

$$
\bar{I}_{x}=L_{21}^{\perp} P_{x}^{* *}+L_{22}^{\perp} E_{x}^{* *},
$$

where $P_{x}^{* *} \equiv\left(\epsilon \zeta_{\mathrm{NS}} / \mu\right) P_{x}, \quad E_{x}^{* *} \equiv\left(\epsilon^{2} \zeta_{\mathrm{NS}}^{2} / \mu L^{2}\right) E_{x}$ are the hydrodynamic and electric forcing parameters with dimensions of current density. The streaming current conductance and the electrical conductance, both dimensionless, are respectively given by

$$
\begin{aligned}
& L_{21}^{\perp}=\hat{A}_{0}\left[\left(\hat{\kappa} \hat{\delta}_{\perp}-\frac{1}{\hat{\kappa} \hat{h}}\right) \tanh (\hat{\kappa} \hat{h})+1\right]+\frac{1}{2} \sum_{n=1}^{M} \hat{A}_{n} \hat{D}_{n} \times\left\{\left(1-\frac{\tanh \left(\hat{\alpha}_{n} \hat{h}\right)}{\hat{\alpha}_{n} \hat{h}}\right)\left[\hat{\beta}_{n} \tanh \left(\hat{\beta}_{n} \hat{h}\right)-\hat{\alpha}_{n} \tanh \left(\hat{\alpha}_{n} \hat{h}\right)\right]\right. \\
& \left.-\tanh \left(\hat{\alpha}_{n} \hat{h}\right)\left[\frac{2 \hat{\alpha}_{n} \hat{\beta}_{n} \tanh \left(\hat{\beta}_{n} \hat{h}\right)-\left(\hat{\beta}_{n}^{2}+\hat{\alpha}_{n}^{2}\right) \tanh \left(\hat{\alpha}_{n} \hat{h}\right)}{\hat{\kappa}^{2} \hat{h}}+\hat{\beta}_{n} \tanh \left(\hat{\beta}_{n} \hat{h}\right) \tanh \left(\hat{\alpha}_{n} \hat{h}\right)-\hat{\alpha}_{n}\right]\right\} \text {, } \\
& L_{22}^{\perp}=\hat{\kappa}^{2} \hat{\sigma}-\frac{\hat{\kappa}^{2} \hat{A}_{0}^{2}}{2}\left[\operatorname{sech}^{2}(\hat{\kappa} \hat{h})+\frac{\tanh (\hat{\kappa} \hat{h})}{\hat{\kappa} \hat{h}}\right]-\frac{\hat{\kappa} \hat{A}_{0} \hat{F}_{0}}{\hat{h}} \tanh (\hat{\kappa} \hat{h})-\frac{\hat{\kappa}^{2}}{4} \sum_{n=1}^{M} \frac{\hat{A}_{n}^{2}}{\left(1-\hat{\alpha}_{n}^{2} / \hat{\beta}_{n}^{2}\right)}\left\{\operatorname{sech}^{2}\left(\hat{\beta}_{n} \hat{h}\right)+\frac{\tanh \left(\hat{\beta}_{n} \hat{h}\right)}{\hat{\beta}_{n} \hat{h}}-2 \frac{\hat{\alpha}_{n}}{\hat{\beta}_{n}} \tanh \left(\hat{\beta}_{n} \hat{h}\right)\right. \\
& \left.\times\left[\frac{\hat{\beta}_{n} \tanh \left(\hat{\beta}_{n} \hat{h}\right)-\hat{\alpha}_{n} \tanh \left(\hat{\alpha}_{n} \hat{h}\right)}{\hat{\alpha}_{n} \hat{\kappa}^{2} \hat{h}^{2}}+\frac{2 \hat{\alpha}_{n} \hat{\beta}_{n} \tanh \left(\hat{\beta}_{n} \hat{h}\right)-\left(\hat{\beta}_{n}^{2}+\hat{\alpha}_{n}^{2}\right) \tanh \left(\hat{\alpha}_{n} \hat{h}\right)}{\hat{\kappa}^{4} \hat{h}^{2}}+\frac{\hat{\beta}_{n} \tanh \left(\hat{\beta}_{n} \hat{h}\right) \tanh \left(\hat{\alpha}_{n} \hat{h}\right)-\hat{\alpha}_{n}}{\hat{\kappa}^{2} \hat{h}}\right]\right\} \\
& -\frac{1}{2 \hat{h}} \sum_{n=1}^{M} \hat{A}_{n} \hat{F}_{n} \times\left\{\left(1-\frac{\tanh \left(\hat{\alpha}_{n} \hat{h}\right)}{\hat{\alpha}_{n} \hat{h}}\right)\left[\hat{\beta}_{n} \tanh \left(\hat{\beta}_{n} \hat{h}\right)-\hat{\alpha}_{n} \tanh \left(\hat{\alpha}_{n} \hat{h}\right)\right]\right. \\
& \left.-\tanh \left(\hat{\alpha}_{n} \hat{h}\right)\left[\frac{2 \hat{\alpha}_{n} \hat{\beta}_{n} \tanh \left(\hat{\beta}_{n} \hat{h}\right)-\left(\hat{\beta}_{n}^{2}+\hat{\alpha}_{n}^{2}\right) \tanh \left(\hat{\alpha}_{n} \hat{h}\right)}{\hat{\kappa}^{2} \hat{h}}+\hat{\beta}_{n} \tanh \left(\hat{\beta}_{n} \hat{h}\right) \tanh \left(\hat{\alpha}_{n} \hat{h}\right)-\hat{\alpha}_{n}\right]\right\}
\end{aligned}
$$

where $\hat{\sigma}$ is the normalized electric conductivity defined in Eq. (31). Equations (63) and (67) are the Onsager relations for the flow and ionic fluxes under transverse forcings. Again, by reciprocity, the non-conjugate coefficients are equal to each other: $L_{12}^{\perp}=L \frac{\perp}{21}$. The proof of this symmetry (not given here) can be performed in a manner similar to that we have presented earlier for the longitudinal flow. The equality of these coefficients by Eqs. (65) and (68) has also been checked numerically.

Also, for the particular cases that we have looked into for the longitudinal flow, exactly analogous results can be deduced for the transverse flow. Without repeating essentially the same details, let us state the results as follows. First, consider the special case when the wall potential is uniform so that $\zeta_{\mathrm{NS}}=\zeta_{\mathrm{S}}$ or $\hat{\zeta}_{\mathrm{S}}=1$. The streaming flow conductance is found to be given by

$$
L_{12}^{\perp}=1+\hat{\kappa} \hat{\delta}_{\perp} \tanh (\hat{\kappa} \hat{h})-\frac{\tanh (\hat{\kappa} \hat{h})}{\hat{\kappa} \hat{h}} \text { for } \quad \hat{\zeta}_{\mathrm{S}}=1
$$

In terms of dimensional quantities, the mean EOF velocity is given by

$$
\begin{aligned}
\bar{u}_{\mathrm{EO}}= & {\left[1+\kappa \delta_{\perp} \tanh (\kappa h)-\frac{\tanh (\kappa h)}{\kappa h}\right] } \\
& \times\left(-\frac{\epsilon \zeta}{\mu} E_{x}\right) \text { for } \zeta_{\mathrm{NS}}=\zeta_{\mathrm{S}}=\zeta .
\end{aligned}
$$

The EOF is enhanced by the slip through the term $\kappa \delta_{\perp} \tanh (\kappa h)$, which is linearly proportional to the effective slip length $\delta_{\perp}$. For transverse flow over perfect-slip slots, the effective slip length is obtainable from Philip ${ }^{21}$ 


$$
\delta_{\perp}=\frac{L}{\pi} \ln \left[\sec \left(\frac{\pi a}{2}\right)\right] \quad \text { for } \quad \lambda=\infty, \hat{h} \gg 1 .
$$

When the stripes are partial slipping, one may use the approximate formula derived by Belyaev and Vinogradova ${ }^{22}$

$$
\delta_{\perp} \simeq \frac{\frac{L}{\pi} \ln \left[\sec \left(\frac{\pi a}{2}\right)\right]}{1+\frac{L}{\pi \lambda} \ln \left[\sec \left(\frac{\pi a}{2}\right)+\tan \left(\frac{\pi a}{2}\right)\right]} \text { for any } \hat{\lambda}, \text { and } \hat{h} \gg 1 \text {. }
$$

Further, for a very thin electric double layer, the EOF velocity in Eq. (71) reduces to

$$
\bar{u}_{\mathrm{EO}}=\left(1+\kappa \delta_{\perp}\right)\left(-\frac{\epsilon \zeta}{\mu} E_{x}\right) \quad \text { for } \quad \kappa h \gg 1,
$$

which matches the transverse component of Eq. (39) derived by Squires. ${ }^{9}$ Hence, in this limit, the EOF is enhanced by a factor equal to the effective slip length divided by the Debye length. Our remarks made earlier for the longitudinal flow apply to the transverse flow as well.

Second, consider the limiting case of a very thin EDL, $\hat{\kappa} \gg 1$, such that $\hat{\beta}_{n} \approx \hat{\kappa}$. Under this limiting condition, the streaming conductance in Eq. (65) approximates to

$$
L_{12}^{\perp} \approx 1+\frac{\hat{\delta}_{\perp}}{\hat{\lambda}}\left[\hat{\zeta}_{S}(1+\hat{\kappa} \hat{\lambda})-1\right] \text { for } \hat{\kappa} \gg 1 .
$$

If the slip surface is perfectly slipping but uncharged, $\hat{\lambda}=\infty$ and $\hat{\zeta}_{\mathrm{S}}=0$, the result further reduces to $L_{12}^{\perp} \approx 1$. In other words, in this limiting case, the EOF velocity is simply equal to the Helmholtz-Smoluchowski velocity without subject to any slip effect

$$
\bar{u}_{\mathrm{EO}}=-\frac{\epsilon \zeta_{\mathrm{NS}}}{\mu} E_{x} \quad \text { for } \quad \zeta_{\mathrm{S}}=0, \quad \hat{\lambda}=\infty, \quad \hat{\kappa} \gg 1
$$

Again, like the longitudinal flow, the transverse EO plug flow is not affected by the uncharged perfect-slipping regions. The remarks made earlier for the longitudinal flow also apply to the transverse flow.

In summary, analogous expressions are obtainable for the macroscopic flow behaviors when the flow is in a principal direction either longitudinal or transverse to the wall pattern.

\section{RESULTS AND DISCUSSIONS}

The input parameters for the computations are the following: the slipping area fraction of the wall $a$, the channel half-height $\hat{h}$, the Debye parameter (or the inverse of the Debye length) $\hat{\kappa}$, the intrinsic or microscopic slip length $\hat{\lambda}$, the zeta potential of the slipping region $\hat{\zeta}_{S}$, and the normalized electric conductivity $\hat{\sigma}$. The coefficients are numerically calculated using Fortran programs, where standard subroutines are called for solving the $M \times M$ systems of equations deduced above. Convergence of the solution is ensured by using a sufficiently large number $(M \geq 300)$ of collocation points to be evenly distributed in the domain $0<\hat{x}<1$.

For applicability of the Boltzmann equation, the EDLs of the two walls should not strongly overlap with each other. Therefore, in the following discussions, we shall limit ourselves to an electric double layer that is of a thickness comparable to or smaller than the channel height or $\hat{\kappa} \hat{h} \geq O(1)$. Very small $\hat{\kappa}$ or thick EDL is excluded in our discussions below.

Let us revisit the kinds of patterns that we have examined in particular in the preceding sections. First, consider the case when the wall is inhomogeneously slipping, but the potential is uniform so that $\zeta_{\mathrm{NS}}=\zeta_{\mathrm{S}}$ or $\hat{\zeta}_{\mathrm{S}}=1$. In this case, the streaming conductance is given by Eq. (37) for longitudinal flow and Eq. (70) for transverse flow. In Figs. 2(a) and 2(c), we show
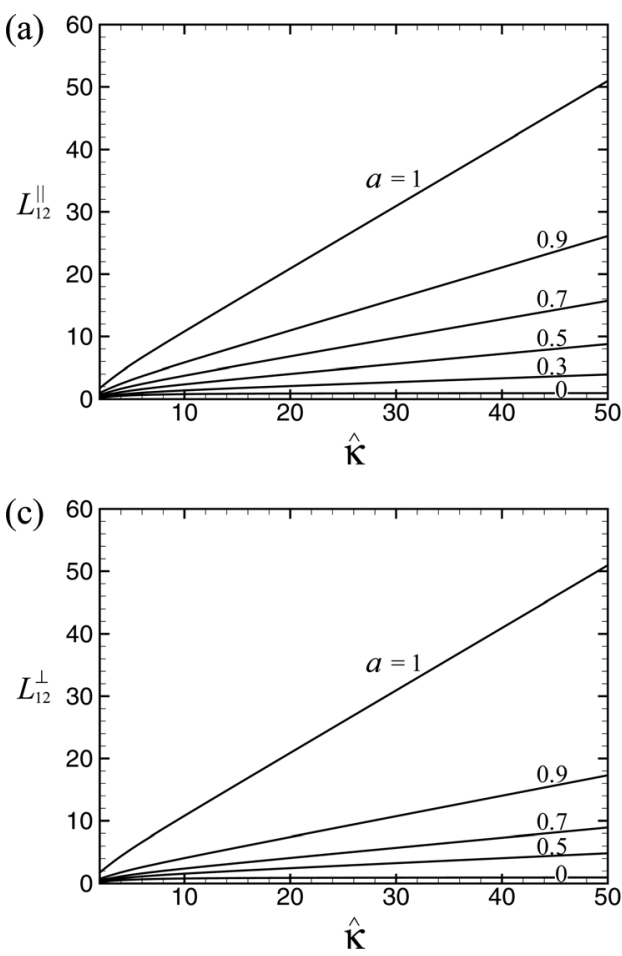
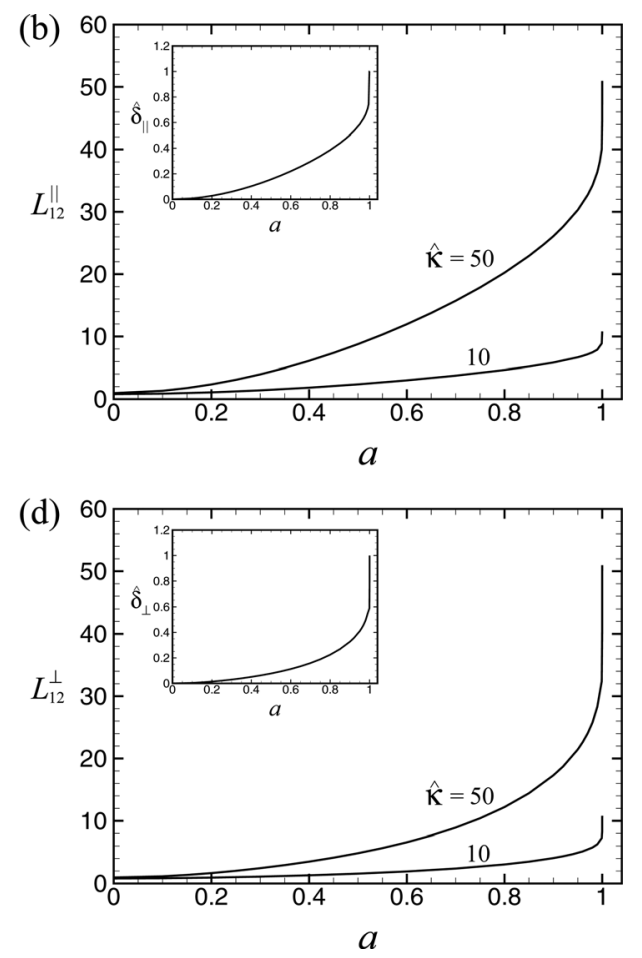

FIG. 2. The longitudinal and transverse streaming conductance, $L_{12}^{\|}$and $L_{12}^{\perp}$, as functions of the Debye parameter $\hat{\kappa}$ and the slipping area of fraction $a$, where the half channel height $\hat{h}=0.5$, intrinsic slip length $\hat{\lambda}=1$, and wall potential $\hat{\zeta}_{\mathrm{S}}=1$. The two insets show the effective slip lengths, $\hat{\delta}_{\|}$and $\hat{\delta}_{\perp}$, as functions of $a$. 
$L_{12}^{\|}$and $L_{12}^{\perp}$ as functions of $\hat{\kappa}$ and $a$, where $\hat{h}=0.5, \hat{\zeta}_{\mathrm{S}}=1$, and $\hat{\lambda}=1$. As $\hat{\kappa}$ increases, the streaming conductance is upper-bounded by the limit of unity in the absence of wall slippage but can increase without bound and almost linearly with $\hat{\kappa}$ in the presence of effective slip, where the rate of increase is larger for larger $a$. As noted earlier, for $\hat{\kappa} \gg 1$, the EO flow is enhanced by a factor equal to the effective slip length divided by the Debye length, which can be very large when the electric double layer is much thinner than the slip length. Here, we see that when the wall is uniformly slipping (i.e., $a=1$ and $\hat{\delta}=\hat{\lambda}$ ), the slip-induced EOF enhancement is indeed large, as given by $\hat{\kappa} \hat{\lambda}$. However, when part of the wall becomes non-slipping (i.e., $a<1$ ), the EOF enhancement can be substantially reduced. It is remarkable that even a very tiny area fraction of the wall becoming non-slipping will materially reduce the effective slip length. There is a sharp decrease of $\hat{\delta}$ as the slipping area fraction $a$ slightly drops below unity; see the insets in Figs. 2(b) and 2(d). This can be confirmed mathematically from Eqs. (41) and (73) for the case of a thick channel. From these equations, one can find that the two effective slip lengths tend to the microscopic slip length $\hat{\lambda}$, while their rate of change with $a$ is asymptotically scaled by $(1-a)^{-1}$, which is very large, as $a$ approaches unity

$$
\begin{aligned}
& \hat{\delta}_{\|, \perp} \rightarrow \hat{\lambda} \quad \text { as } \quad a \rightarrow 1^{-}, \\
& \frac{\mathrm{d} \hat{\delta}_{\|}}{\mathrm{d} a} \rightarrow \frac{\frac{2}{\pi(1-a)}}{\left[\frac{2}{\pi \hat{\lambda}} \ln \left(\frac{4 / \pi}{1-a}\right)+1\right]} \\
& -\frac{\frac{4}{\pi^{2} \hat{\lambda}(1-a)} \ln \left(\frac{2 / \pi}{1-a}\right)}{\left[\frac{2}{\pi \hat{\lambda}} \ln \left(\frac{4 / \pi}{1-a}\right)+1\right]^{2}} \quad \text { as } \quad a \rightarrow 1^{-} \text {, } \\
& \frac{\mathrm{d} \hat{\delta}_{\perp}}{\mathrm{d} a} \rightarrow \frac{\frac{1}{\pi(1-a)}}{\left[\frac{1}{\pi \hat{\lambda}} \ln \left(\frac{4 / \pi}{1-a}\right)+1\right]} \\
& -\frac{\frac{1}{\pi^{2} \hat{\lambda}(1-a)} \ln \left(\frac{2 / \pi}{1-a}\right)}{\left[\frac{1}{\pi \hat{\lambda}} \ln \left(\frac{4 / \pi}{1-a}\right)+1\right]^{2}} \text { as } a \rightarrow 1^{-} \text {. }
\end{aligned}
$$

Hence, the very large rate of change will cause the effective slip lengths to drop significantly as the slipping area fraction drops slightly from unity. Consequently, there is a corresponding sharp decrease of the EOF enhancement when the wall condition deviates slightly from 100\% slipping; see Figs. 2(b) and 2(d). At $\hat{\kappa}=50$, the longitudinal streaming conductance $L_{12}^{\|}$drops by $28 \%$ when $a$ barely decreases from unity to 0.99 . The transverse streaming conductance $L_{12}^{\perp}$ drops by an even larger amount of $44 \%$ for the same minute change of $a$. Our conclusion here is that the slipinduced EOF enhancement (which is also interpreted as slipenhanced apparent zeta potential) can be dramatically reduced by a small fraction (as small as 1\%) of the wall being covered by periodically distributed non-slipping stripes. Should just a small fraction of a slipping surface be contaminated by non-slipping slots, the mistake will be large if one ignores such sensitivity and estimates the EO flow based on the assumption that the wall slippage is perfectly uniform. Estimation based on small perturbations does not work at the limit $a=1$. Also note that because of the anisotropic effective slip $\hat{\delta}_{\|}>\hat{\delta}_{\perp}$, the EO flow enhancement due to the hydrodynamic slip is always larger in the longitudinal direction than in the transverse direction, $L_{12}^{\|}>L_{12}^{\perp}$, for fixed $\hat{\kappa}$ and $0<a<1$.

Second, we examine the case when alternate stripes are perfectly slipping but uncharged: $\hat{\lambda}=\infty$ and $\hat{\zeta}_{\mathrm{S}}=0$, and when the electric double layer is extremely thin: $\hat{\kappa} \gg 1$. A formal proof of Squires ${ }^{9}$ has led him to conclude that this kind of surface (uncharged where it is perfectly slipping, and charged where it is non-slipping) will not give rise to any EO flow enhancement due to the slip, instead giving precisely the same EO flow as if the surface were completely non-slipping and homogeneously charged. Let us now look into the range of validity of this theoretical limit. In Figs. 3(a) and 3(c), we show $L_{12}^{\|}$and $L_{12}^{\perp}$ as functions of $\hat{\kappa}$ and $a$, where $\hat{h}=0.5, \hat{\zeta}_{\mathrm{S}}=0$ and $\hat{\lambda}=\infty$. Indeed, for very large Debye parameter $\hat{\kappa}>O\left(10^{3}\right)$, either coefficient will tend to unity for any slipping area fraction $0 \leq a<1$. In other words, for extremely thin electric double layers, the perfectslipping-uncharged regions will indeed have negligible effect on the EO flow, which is then approximately given by the Helmholtz-Smoluchowski limit. However, for order unity to moderately large Debye parameter $\hat{\kappa}=O\left(1-10^{3}\right)$, it is clear from the figures that the perfect-slipping-uncharged regions can have finite effects on the EO flow. The effect is, however, not enhancement, but inhibition of the EO flow. The flow is reduced because of decrease in the charged wall area, which outweighs any enhancement due to increase in the slipping wall area. The effect is larger for larger $a$ at a fixed value of $\hat{\kappa}$. Figures 3(b) and 3(d) show how the two coefficients $L_{12}^{\|}$and $L_{12}^{\perp}$ will decrease with increasing $a$ for $\hat{\kappa}=1000,50$. At $\hat{\kappa}=1000$, the coefficients remain close to unity until $a$ becomes larger than 0.9 . The coefficients drop abruptly toward zero on approaching the limit of a completely uncharged wall, $a=1$. For a smaller $\hat{\kappa}=50$, the coefficients deviate from the non-slipping limit at a larger rate with increasing $a$. When $a$ increases to 0.9 , the longitudinal coefficient $L_{12}^{\|}$drops by $20 \%$, while the transverse coefficient $L_{12}^{\perp}$ drops by a larger amount of $30 \%$. Hence, the flow is more affected when it is normal to the stripes. Our conclusion here is that slipping-uncharged regions on a surface will have practically no effect on the EO flow only when the normalized Debye parameter is very large, $\hat{\kappa}>O\left(10^{3}\right)$. When $\hat{\kappa}$ is only moderately large, the decreasing effect due to the slipping-uncharged regions should not be neglected.

We further examine uncharged stripes, $\hat{\zeta}_{\mathrm{S}}=0$, but with a finite slip length, $\hat{\lambda}=1$. From Eqs. (44) and (75), the thin EDL limit is

$$
L_{12}^{\|, \perp} \approx 1-\frac{\hat{\delta}_{\|, \perp}}{\hat{\lambda}} \quad \text { for } \quad \hat{\kappa} \gg 1 .
$$


(a)

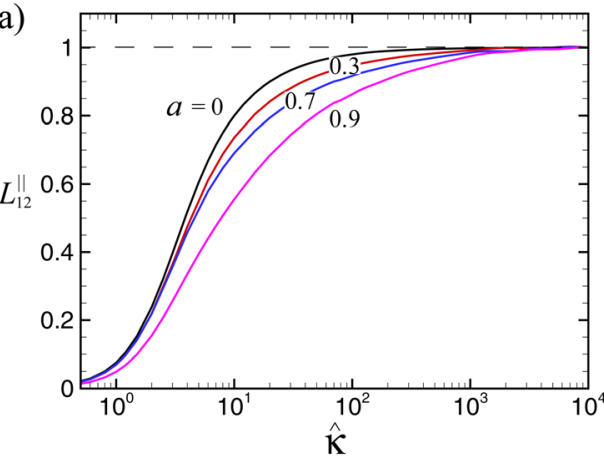

(c)

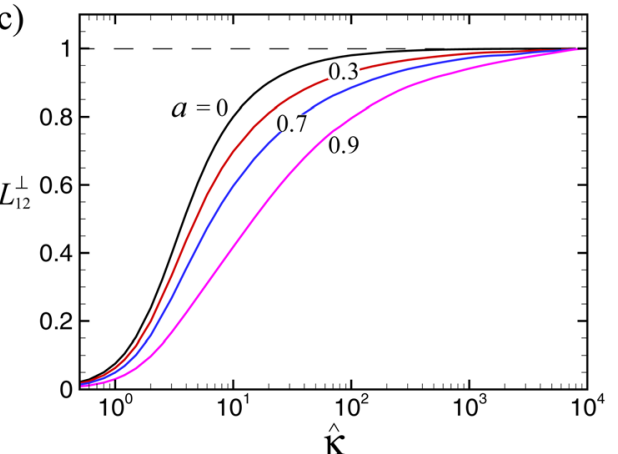

(b)

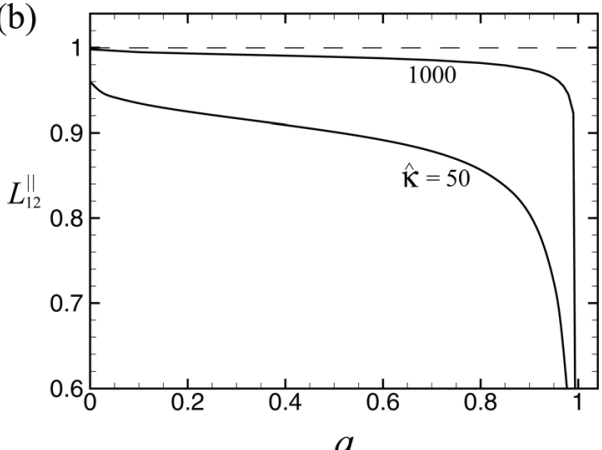

(d)

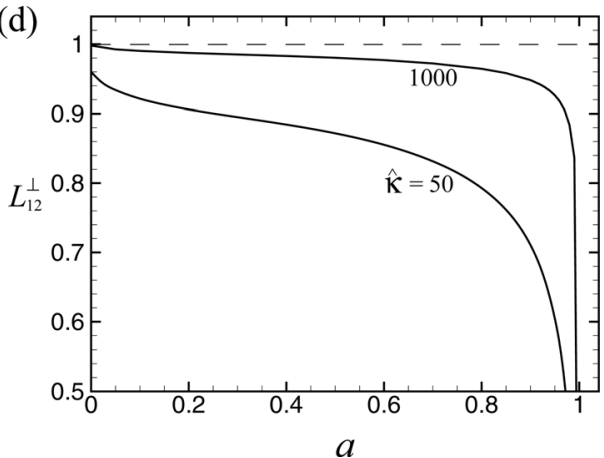

FIG. 3. (Color online) The longitudinal and transverse streaming conductance, $L_{12}^{\|}$and $L_{12}^{\perp}$, as functions of the Debye parameter $\hat{\kappa}$ and the slipping area of fraction $a$, where the half channel height $\hat{h}=0.5$, intrinsic slip length $\hat{\lambda}=\infty$, and wall potential $\hat{\zeta}_{\mathrm{S}}=0$.
Let us also look into the range of validity of this theoretical limit. In Figs. 4(a) and 4(b), we show $L_{12}^{\|}$and $L_{12}^{\perp}$ as functions of $\hat{\kappa}$ and $a$, where $\hat{h}=0.5, \hat{\zeta}_{\mathrm{S}}=0$, and $\hat{\lambda}=1$. Here, we see that when the partial-slipping regions are uncharged, the EO flow is always inhibited by the presence of these slipping regions, as has been pointed out by Belyaev and Vinogradova. ${ }^{15}$ Again, it requires the Debye parameter to be as large as $\hat{\kappa}>O\left(10^{3}\right)$ for the coefficients to get close to the upper limit given by Eq. (80). The thin EDL limit should not be used for $\hat{\kappa}<10^{3}$, especially for the transverse case. Note that Fig. 4 shows a case of anisotropy that is in contrast to common expectations. ${ }^{15}$ The transverse coefficient is larger than the longitudinal coefficient: $L_{12}^{\perp}>L_{12}^{\|}$, as a result of the fact that $\hat{\delta}_{\|}>\hat{\delta}_{\perp}$ for $0<a<1$.

We next examine the case when the stripes are perfectly slipping, $\hat{\lambda}=\infty$, but are very weakly charged, $\hat{\zeta}_{\mathrm{S}}=0.01$. In this case, the thin EDL limit is

$$
L_{12}^{\|, \perp} \approx 1+\hat{\zeta}_{S} \hat{\kappa} \hat{\delta}_{\|, \perp} \quad \text { for } \quad \hat{\kappa} \gg 1
$$

Figures 5(a) and 5(b) show $L_{12}^{\|}$and $L_{12}^{\perp}$ as functions of $\hat{\kappa}$ and $a$, where $\hat{h}=0.5, \hat{\zeta}_{\mathrm{S}}=0.01$, and $\hat{\lambda}=\infty$. The weak surface potential of the slipping regions will have negligible effect on the coefficients for sufficiently low $\hat{\kappa}$ such that $\hat{\zeta}_{\mathrm{S}} \hat{\kappa} \leq O(1)$, where the EO flow is retarded by the hydrodynamic slip. The trend will dramatically change when the Debye parameter becomes sufficiently large, say $\hat{\zeta}_{\mathrm{S}} \hat{\kappa}>O(1)$, for which the EO flow will be significantly enhanced by the presence of the slipping regions with even a very small surface potential. Here, the anisotropy is as usual: $L_{12}^{\|}>L_{12}^{\perp}$ for fixed $\hat{\kappa}$ and $0<a<1$.

Finally, let us examine how the present stick-slip wall pattern may affect electrokinetic energy conversion. Mechanical energy can be converted into electrical energy in a microchannel when streaming potential and streaming current are generated under an applied pressure difference between the ends of the channel. ${ }^{28}$ The conversion efficiency at the maximum generation power is given by Xuan and $\mathrm{Li}^{28}$ to be $\eta_{\max W}=Z /[2(2-Z)]$, where $Z$ in the present notation is

$$
Z=\frac{L_{12} L_{21}}{L_{11} L_{22}},
$$

(a)

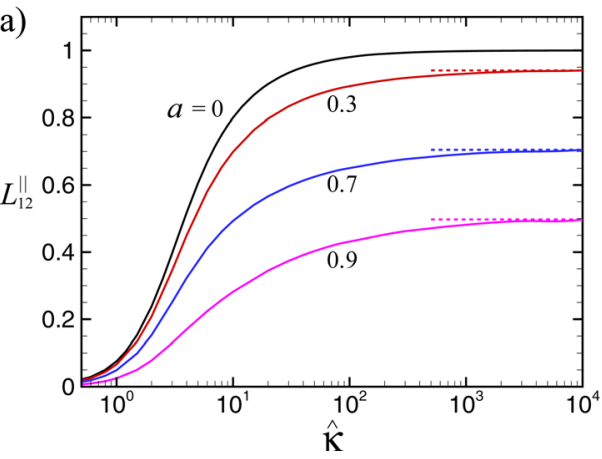

(b)

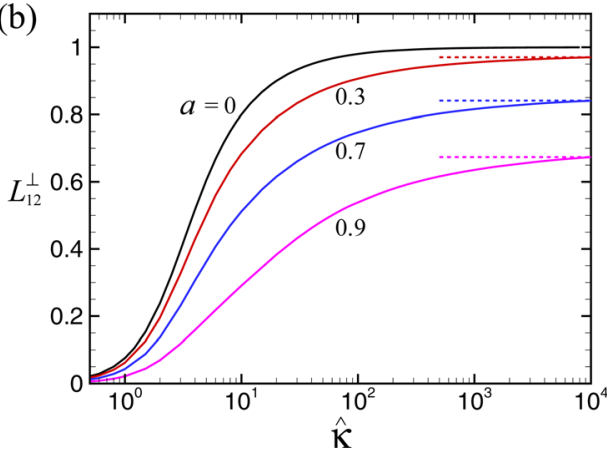

FIG. 4. (Color online) The longitudinal and transverse streaming conductance, $L_{12}^{\|}$and $L_{12}^{\perp}$, as functions of the Debye parameter $\hat{\kappa}$ and the slipping area of fraction $a$, where the half channel height $\hat{h}=0.5$, intrinsic slip length $\hat{\lambda}=1$, and wall potential $\hat{\zeta}_{\mathrm{s}}=0$. The dashes are the thin EDL limits for $\hat{\kappa} \gg 1$ given by Eq. (80). 

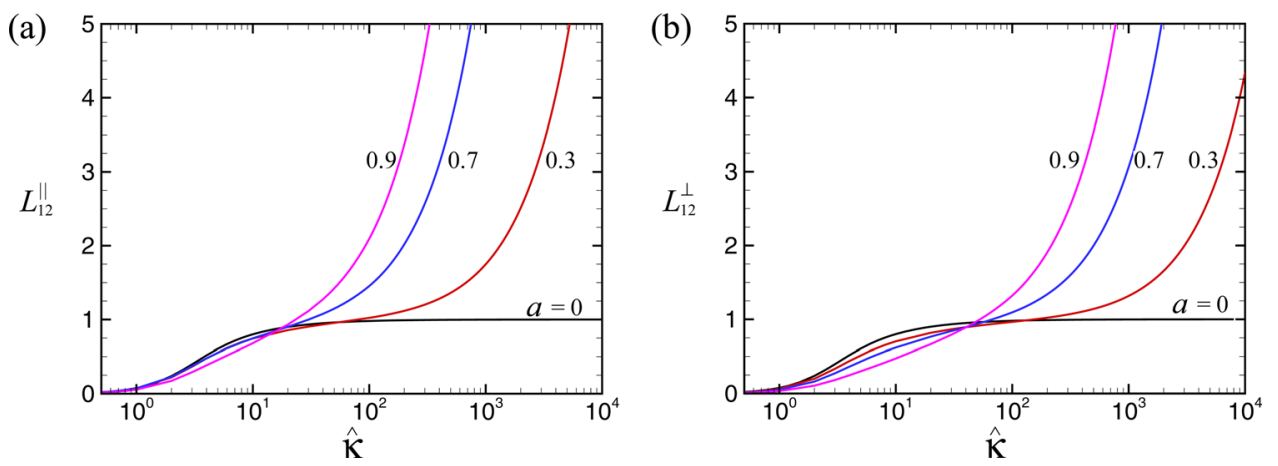

FIG. 5. (Color online) The longitudinal and transverse streaming conductance, $L_{12}^{\|}$and $L_{12}^{\perp}$, as functions of the Debye parameter $\hat{\kappa}$ and the slipping area of fraction $a$, where the half channel height $\hat{h}=0.5$, intrinsic slip length $\hat{\lambda}=\infty$, and wall potential $\hat{\zeta}_{\mathrm{S}}=0.01$. Note that the curves tend to the thin EDL limits for $\hat{\kappa} \gg 1$ given by Eq. (81).

which is called the figure of merit of the device. Obviously, the efficiency increases monotonically with $Z$, and therefore it is desirable to achieve as large the figure of merit as possible. Some recent studies have shown that electrokinetic energy conversion can achieve a higher efficiency under the effect of wall slip. ${ }^{29,30}$ Let us find out how the figure of merit will be affected by the wall pattern under consideration. In Figs. 6(a) and 6(c), we show $Z_{\|}$and $Z_{\perp}$ as functions of $\hat{\kappa}$ and $a$, where $\hat{h}=0.5, \hat{\zeta}_{\mathrm{S}}=1, \hat{\lambda}=1$, and $\hat{\sigma}=0.5$. Our observations are as follows. First, the figure of merit is clearly enhanced by wall slip. The larger the slipping area fraction $a$, the larger the figure of merit $Z$. Also, for given $a$, the figure of merit will attain a maximum value at a particular value of $\hat{\kappa}$, which varies depending on $\hat{h}$ and $\hat{\sigma}$. Near the peak, the enhancement due to slip is more than a factor of 2 between the limiting cases $a=1$ and $a=0$. Our results are qualitatively consistent with the results reported previously by Davidson and Xuan. ${ }^{29}$ Second, in the absence of wall slip, the figure of merit drops to practically zero at $\hat{\kappa} \gg 1$. With wall slip, the figure of merit maintains a positive finite value even when $\hat{\kappa}$ becomes large. Third, as shown in Figs. 6(b) and 6(d), for any given $0<a<1$, the figure of merit is larger when the flow is longitudinal than when it is transverse. Hence, it is more advantageous to orient the flow along the stripes for a better energy conversion efficiency. Fourth, we see again the sharp decline of the quantities as $a$ drops slightly from unity. The extent of sharp decline is larger for transverse flow than longitudinal flow. One has to be cautious about this sensitivity on estimating the enhancement effect due to slip on the figure of merit.

\section{CONCLUDING REMARKS}

In this paper, we have deduced the Onsager relations for electroosmotic (EO) flow through a parallel-plate channel, of which the walls are patterned with periodic stripes of alternating slip lengths $(0, \lambda)$ and alternating zeta potentials $\left(\zeta_{\mathrm{NS}}, \zeta_{\mathrm{S}}\right)$. Our model is more general than existing ones in the literature as it can handle finite channel height, arbitrary electric double layer thickness, and also partial slip length

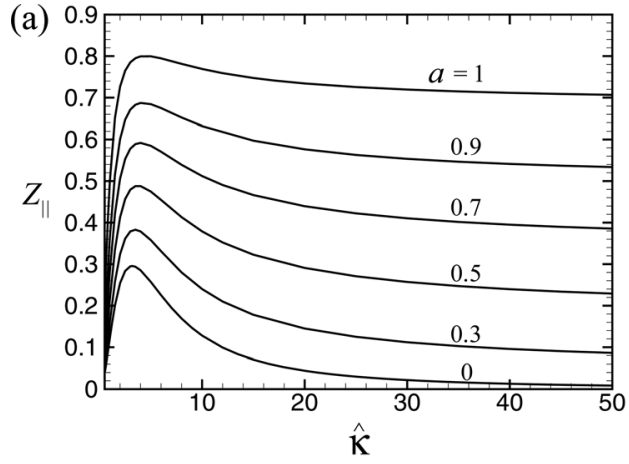

(c)

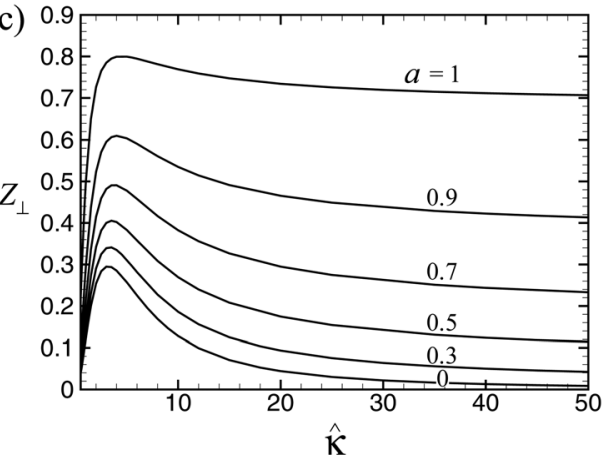

(b)

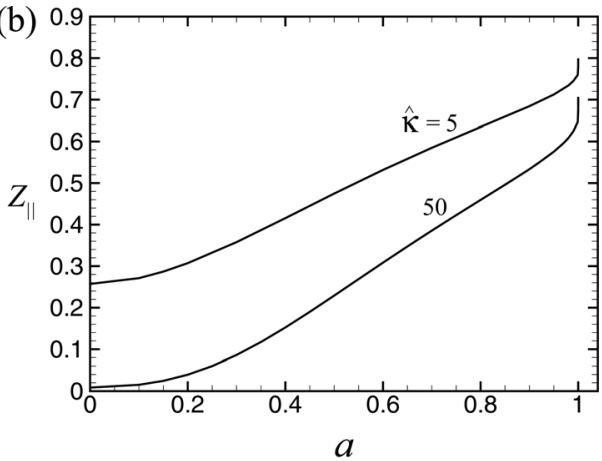

(d)

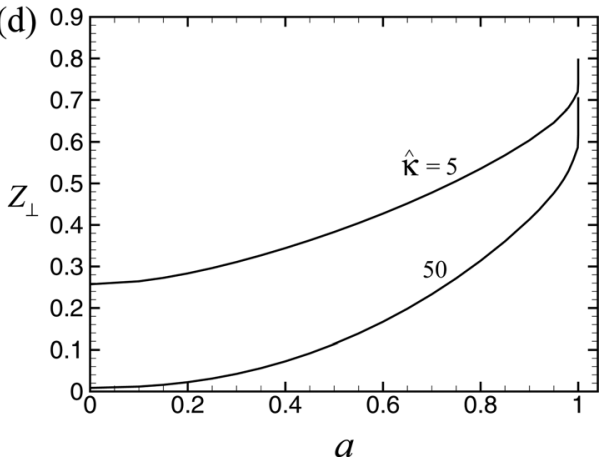

FIG. 6. The longitudinal and transverse figures of merit, $Z_{\|}$and $Z_{\perp}$, as functions of the Debye parameter $\hat{\kappa}$ and the slipping area of fraction $a$, where the half channel height $\hat{h}=0.5$, intrinsic slip length $\hat{\lambda}=1$, and wall potential $\hat{\zeta}_{\mathrm{s}}=1$. 
$0 \leq \lambda \leq \infty$. Flows in the principal directions, i.e., parallel and normal to the stripes, have been considered. Flow that is oriented at any other angle with respect to the pattern can be readily deduced following, e.g., Ajdari. ${ }^{31}$

We have formally proved the reciprocal symmetry of the Onsager relations for EO flow over a striped superhydrophobic surface. We have also deduced analytical results in agreement of those in the literature ${ }^{9,12,14,15}$ for some particular kinds of stripe patterns. When the wall is uniformly charged, $\zeta_{\mathrm{NS}}=\zeta_{\mathrm{S}}$, the effective slip length obtained from the hydrodynamic problem can be used directly in the EO flow as if the wall were uniformly slipping with slippage equal to the effective slip length. We have shown that this result is true for any values of $\lambda$ and the Debye parameter $\hat{\kappa}$. For sufficiently large $\hat{\kappa}$, the EOF enhancement factor due to slip is well known to be the effective slip length multiplied by the Debye parameter. We point out that as shown by our numerical results, this enhancement factor can be dramatically reduced when as small as $1 \%$ by area of an otherwise uniformly slipping wall is contaminated by periodically distributed no-slip slots.

EO flow is theoretically shown to be unaffected by wall regions which are perfectly slipping but uncharged, under the condition of very thin electric double layers. Our numerical results reveal that this theoretical limit is practically achieved only when the Debye parameter is as large as $\hat{\kappa}>O\left(10^{3}\right)$. Any smaller Debye parameter will see a decreasing effect of the slipping-uncharged regions on the EO flow, essentially because of a decrease in the charged wall area. Uncharged partial-slipping stripes will always inhibit the EO flow. The larger the slipping area fraction, the larger the inhibition, as long as the slipping area is uncharged. However, should the slipping regions become only slightly charged, the inhibition trend will be dramatically reversed, and the EO flow will be appreciably enhanced for sufficiently large Debye parameter $\hat{\kappa}$. We have also shown how the wall pattern may have effect on the figure of merit of the channel when it is used for electrokinetic energy conversion.

\section{ACKNOWLEDGMENTS}

Financial support was given by the Research Grants Council of the Hong Kong Special Administrative Region, China, through Project Nos. HKU 715609E and HKU $715510 \mathrm{E}$, and also by the University of Hong Kong through the Seed Funding Programme for Basic Research under Project Code 200911159024. Private communications with Professor Martin Z. Bazant and Professor Olga I. Vinogradova, and comments by the referees are gratefully acknowledged.

${ }^{1}$ R. F. Probstein, Physicochemical Hydrodynamics (Wiley, New York, 1994).

${ }^{2}$ D. Li, Electrokinetics in Microfluidics (Elsevier, Oxford, 2004).

${ }^{3}$ S. Kandlikar, S. Garimella, D. Li, S. Colin, and M. R. King, Heat Transfer and Fluid Flow in Minichannels and Microchannels (Elsevier, Oxford, 2006).
${ }^{4}$ J. H. Masliyah and S. Bhattacharjee, Electrokinetic and Colloid Transport Phenomena (Wiley, New Jersey, 2006).

${ }^{5}$ J. P. Rothstein, "Slip on superhydrophobic surfaces," Annu. Rev. Fluid Mech. 42, 89 (2010).

${ }^{6}$ N. V. Churaev, J. Ralston, I. P. Sergeeva, and V. D. Sobolev, "Electrokinetic properties of methylated quartz capillaries," Adv. Colloid Interface Sci. 96, 265 (2002).

${ }^{7}$ L. Joly, C. Ybert, E. Trizac, and L. Bocquet, "Liquid friction on charged surfaces: From hydrodynamic slippage to electrokinetics," J. Chem. Phys. 125, 204716 (2006).

${ }^{8} \mathrm{~V}$. Tandon and B. J. Kirby, "Zeta potential and electroosmotic mobility in microfluidic devices fabricated from hydrophobic polymers: 2. Slip and interfacial water structure," Electrophoresis 29, 1102 (2008).

${ }^{9}$ T. M. Squires, "Electrokinetic flows over inhomogeneously slipping surfaces," Phys. Fluids 20, 092105 (2008).

${ }^{10} \mathrm{~W}$. Qu and D. Li, "A model for overlapped EDL fields," J. Colloid Interface Sci. 224, 397 (2000).

${ }^{11} \mathrm{~F}$. Baldessari, "Electrokinetics in nanochannels Part I. Electric double layer overlap and channel-to-wall equilibrium," J. Colloid Interface Sci. 325, 526 (2008).

${ }^{12}$ S. S. Bahga, O. I. Vinogradova, and M. Z. Bazant, "Anisotropic electroosmotic flow over super-hydrophobic surfaces," J. Fluid Mech. 644, 245 (2010).

${ }^{13}$ H. Zhao, "Electro-osmotic flow over a charged superhydrophobic surface," Phys. Rev. E 81, 066314 (2010).

${ }^{14} \mathrm{H}$. Zhao, "Streaming potential generated by a pressure-driven flow over superhydrophobic stripes," Phys. Fluids 23, 022003 (2011).

${ }^{15} \mathrm{~A}$. V. Belyaev and O. I. Vinogradova, "Electro-osmosis on anisotropic superhydrophobic surfaces," Phys. Rev. Lett. 107, 098301 (2011).

${ }^{16}$ S. S. Dukhin, "Non-equilibrium electric surface phenomena," Adv. Colloid Interface Sci. 44, 1 (1993).

${ }^{17} \mathrm{C}$. O. Ng and C. Y. Wang, "Stokes shear flow over a grating: Implications for superhydrophobic slip," Phys. Fluids 21, 013602 (2009).

${ }^{18} \mathrm{C}$. O. Ng, H. C. W. Chu, and C. Y. Wang, "On the effects of liquid-gas interfacial shear on slip flow through a parallel-plate channel with superhydrophobic grooved walls," Phys. Fluids 22, 102002 (2010).

${ }^{19}$ C. O. Ng and C. Y. Wang, "Stokes flow through a periodically grooved tube,” ASME J. Fluids Eng. 132, 101204 (2010).

${ }^{20}$ C. O. $\mathrm{Ng}$ and C. Y. Wang, "Oscillatory flow through a channel with stickslip walls: Complex Naviers slip length,” ASME J. Fluids Eng. 133, 014502 (2011).

${ }^{21}$ J. R. Philip, "Flows satisfying mixed no-slip and no-shear conditions," ZAMP 23, 353 (1972).

${ }^{22}$ A. V. Belyaev and O. I. Vinogradova, "Effective slip in pressure-driven flow past super-hydrophobic stripes," J. Fluid Mech. 652, 489 (2010).

${ }^{23}$ L. Joly, C. Ybert, E. Trizac, and L. Bocquet, "Hydrodynamics within the electric double layer on slipping surfaces," Phys. Rev. Lett. 93, 257805 (2004).

${ }^{24} \mathrm{~A}$. Ajdari and L. Bocquet, "Giant amplification of interfacially driven transport by hydrodynamic slip: Diffusio-osmosis and beyond," Phys. Rev. Lett. 96, 186102 (2006).

${ }^{25}$ C. I. Bouzigues, P. Tabeling, and L. Bocquet, "Nanofluidics in the Debye layer at hydrophilic and hydrophobic surfaces," Phys. Rev. Lett. 101, 114503 (2008).

${ }^{26}$ O. I. Vinogradova and A. V. Belyaev, "Wetting, roughness and flow boundary conditions," J. Phys.: Condens. Matter 23, 184104 (2011).

${ }^{27}$ D. M. Huang, C. Cottin-Bizzone, C. Ybert, and L. Bocquet, "Massive amplification of surface-induced transport at superhydrophobic surfaces," Phys. Rev. Lett. 101, 064503 (2008).

${ }^{28} \mathrm{X}$. Xuan and D. Li, "Thermodynamic analysis of electrokinetic energy conversion,” J. Power Source 156, 677 (2006).

${ }^{29} \mathrm{C}$. Davidson and X. Xuan, "Electrokinetic energy conversion in slip nanochannels," J. Power Source 179, 297 (2008).

${ }^{30}$ Y. Ren and D. Stein, "Slip-enhanced electrokinetic energy conversion in nanofluidic channels," Nanotechnology 19, 195707 (2008).

${ }^{31}$ A. Ajdari, "Transverse electrokinetic and microfluidic effects in micropatterned channels: Lubrication analysis for slab geometries," Phys. Rev. E 65, 016301 (2001). 